\title{
Ulrich Herrmann
}

\section{"ungenau in dieser Welt" - kein Krawall, kein Protest: Der unaufhaltsame Aufstieg um 1940 Geborener in einer „Generationen“-Lücke}

\author{
... das Jahrhundert, als welches sowohl den Willigen als \\ Unwilligen mit sich fortreißt, bestimmt und bildet, dergestalt, daß \\ man wohl sagen kann, ein jeder, nur zehn Jahre früher oder später \\ geboren, dürfte, was seine eigene Bildung und die Wirkung nach \\ außen betrifft, ein ganz anderer geworden sein. \\ Goethe, Dichtung und Wahrheit, I. Teil, Einleitung
}

Andreas Flitner, geb. 1922, "Kriegsgeneration“, zum 80. Geburtstag gewidmet

Der vorliegende Beitrag soll erstens zur Klärung eines empirisch gehaltvollen und analytisch präzisierten Begriffs von "Generation" beitragen; zweitens den Versuch machen, wie man all jene Individuen und Gruppen sozial und geschichtlich einordnen könne, die sich selber gar keiner "Generation" zurechnen und auch von außen keiner zugeschrieben werden; drittens diese näher zu charakterisieren und dabei auch autobiographische Züge nicht zu vermeiden. Schließlich soll die Verbindung zu den anderen Konferenz-Beiträgen hergestellt werden. Dies erklärt die vorliegende Textgestalt.

Kritisch gelesen und ausführlich kommentiert wurde der Text von Wolfgang Kraushaar (Hamburg). Seine Kritikpunkte, Anregungen und Hinweise sind im Text berücksichtigt worden. Sie lassen auch eine Vorbemerkung geraten erscheinen, ohne die dieser Beitrag leicht mißverstanden werden könnte: Es handelt sich nicht um den Versuch - wie auch unten mehrfach begründet und erklärt -, eine neue "Generation" konstruieren zu wollen, sondern um den Versuch der Charakterisierung einer Generations- „Lagerung “ (das wird unten erklärt) von Angehörigen benachbarter Jahrgänge und ihrer Ausgangslage in Kindheit und Jugend in einem bestimmten westdeutschen Nachkriegsmilieu, die sich nicht in Formen kollektiven Handelns gesellschaftlich bemerkbar machten oder plazierten. Sie lassen sich gut unterscheiden von anderen Generations- ${ }_{n}$ Einheiten “ mit ähnlicher Ausgangslage in Westdeutschland, die später aber in ganz anderer Weise politisch aktiv wurden. Kraushaar wies darauf hin, daß sich der „harte Kern“ der 68er-Bewe- 
gung ebenfalls aus den Jahrgängen um 1940 speist. Um diese Differenzierung sichtbar machen zu können, mußten die analytischen begrifflichen Instrumente bereitgelegt werden. Die Frage war sodann: Wie läßt sich zwischen den diskontinuierlichen Aktivitäten von Generations- „Einheiten “ - das ist mit "Lücke gemeint - die zumeist unbemerkte, jedenfalls unspektakuläre „Normalität“ von Lebenswegen in einer Generations- „Lagerung " verstehen, analysieren und in ihrer Bedeutung bewerten? Lohnt sich das überhaupt ${ }^{l}$ ? Wir beginnen mit zwei Untersuchungen, die ähnlichen Phänomenen auf der Spur waren.

\section{Die "Generation der Unbefangenen“}

Von Viggo Graf Blücher erschien 1966 die analytische Darstellung der Ergebnisse einer Emnid-Untersuchung unter dem Titel „Die Generation der Unbefangenen “2. Im gesamten Bundesgebiet waren 2380 Interviews mit jungen Leuten im Alter von 15 bis unter 25 Jahren durchgeführt worden. Jugend und Beruf, die Beziehungen junger Menschen zu ihren Familien, "Gesellungsweisen“ im Jugendalter, Freizeit und Urlaub, Geld, Besitz und Konsum und schließlich Öffentlichkeit, Politik und Gesellschaft waren die Themen der Befragung und der Auswertung und bilden den Hintergrund der allgemein formulierten Befunde. Das Durchschnittsalter der Befragungsgruppe betrug (im Januar 1964) knapp 20 Jahre ${ }^{3}$. Bemerkenswert war, daß durch die Struktur der Stichprobe eine Repräsentation der Jahrgänge 1940-49 gelungen war und daß sich demzufolge das Befragungsergebnis verallgemeinern lie $\beta^{4}$.

Die Befragungsergebnisse und die interpretierenden Schlußfolgerungen der Forschergruppe hatten, wie nicht anders zu erwarten, ein anderes inhaltiches Profil als die Befunde und Schlußfolgerungen von Helmut Schelsky in seinem Buch „Die skeptische Generation“5, einem jugendpsychologischen und -soziologischen Bestseller - weil er einer Vätergeneration den Habitus einer überlebt ha-

${ }^{1}$ Heinz Bude (Berlin) fragte denn auch nicht ohne Grund in der Diskussion, ob solche Lebenswege in solchen Gruppierungen für den Betrachter nicht eigentlich „langweilig " wären? Gewiß, sie können nicht ohne weiteres auf das Interesse des Chronisten oder der Gegenwartsgeschichtsschreibung rechnen - aber das ist die Perspektive der nachgeborenen Betrachier.

2 Viggo Graf Blücher, Die Generation der Unbefangenen. Zur Soziologie der jungen Menschen heute. Unter Mitarbeit von Detlef Kantowsky (Düsseldorf, Köln 1966) 20; im folgenden zitiert: Blücher, Generation.

3 Ebd., 15, 404.

4 Ebd., 15, die Daten $404 \mathrm{f}$.

${ }^{5}$ Helmut Sichelsky, Die skeptische Generation. Eine Soziologie der deutschen Jugend (Düsseldorf 1957, zahlr. Aufl., Frankfurt 1984 mit einem Nachwort des Vf.). Schelsky untersucht die berufstätige Jugend zwischen 14 und 25 im Nachkriegsjahrzehnt 1945-1955. Zur Publikations- und Wirkungsgeschichte dieses Buches vgl. jetzt die instruktive Studie von Franz-Werner Kersting, Helmut Schelskys "Skeptische Generation“ von 1957, in: Vierteljahreshefte für Zeitgeschichte 50 (2002) 465-495. 
benden Söhnegeneration (der 1930 und später Geborenen) erklärte -, ein Buch, dem Kenner der Jugendgeschichte wie Helmut Fend auch heute noch eine erstaunliche Deutungskraft bescheinigen'. Die „skeptische Generation “ umfaßt jene Geburtsjahrgänge, deren Erleben und Bewußtseinsbildung in ihrer Jugendzeit, zum Teil noch in der Kindheit, durch Kriegsereignisse, Luftangriffe und Gefangenschaft, Flucht und Vertreibung und Nachkriegselend geprägt worden waren, durch den Zerfall von Lebens- und Wertordnungen, durch die unmittelbare Lebensnot und das Problem, irgendwo und irgendwie die Schule zu beenden und eine Ausbildung beginnen zu können. Enttäuschung durch die Erlebnisse der vergangenen Jahre, Unsicherheit in der Gegenwart, Skepsis im Hinblick auf die Zukunft - dies kennzeichnete das Bewußtsein der Angehörigen der "skeptischen Generation“, häufig genug ihr Leben lang. Die „skeptische Generation“ konzentrierte sich auf das Fortkommen im Beruf und die Gestaltung des Privatlebens. Politik war bei den "gebrannten Kindern“ nicht gefragt ${ }^{7}$.

Dagegen sah die Mentalität der Jahrgänge 1939-1949, die Blücher und sein Team untersuchten, sehr anders aus ${ }^{8}$ : „Nur in den ältesten Jahrgängen haben diese jungen Leute noch ein wenig vom Krieg und der Nachkriegszeit bewußt erlebt. In die Berufsentwicklung sind sic 1955 eingetreten, in einer Zeit also, in der es schon eine recht große Freiheit der Berufswahl gab. Die persönlichkeitsbildende Zeit haben diese Jugendlichen zwischen 1953 und 1960 erlebt, d. h. in einer Welt, die von Jahr zu Jahr ,normaler' wurde und in der sich der Wohlstand ständig ausbreitete. Diese Jugend kennt in ihrer ganzen Lebenserfahrung immer nur eine Verbesserung ihrer Situation. "9 Aus dieser Lebenserfahrung erwuchs ein Habitus, den die Jugendsoziologen folgendermaßen beschrieben haben: „Das Interesse ist wach, die Unterrichtung geschieht aus den vielfältigsten Quellen. Ein Engagement ist bei kleinen Teilgruppen stark ausgeprägt, die Masse verharrt in passiver Interessiertheit. Betontes Desinteresse betrifft nur unbedeutende Mindergruppen. ... und nach dem Skeptizismus der enttäuschten Nachkriegsjugend findet sich hier erstmals wieder eine Generation, die als, völlig normal' zu bezeichnen man allen Grund hat. ${ }^{\text {}} 10$

Und warum können diese jungen Leute als "unbefangen“ bezeichnet werden? „Es fehlen bei dieser [westdeutschen] Jugend alle ideologischen Fixierungen und dementsprechend auch - die grundsätzliche Ablehnung der Ideologie (die ihrerseits Ideologie ist). Ebenso fehlen Fixierungen an starke weltanschauliche Bindungen wie Religion, Vaterland, Europa. Wohin wir blicken: Pluralismus, Vielfalt, Offenheit, partielles Engagement, Weltneugier, Vorurteilslosigkeit - Unbefangenheit

- Helmut Fend, Sozialgeschichte des Aufwachsens. Bedingungen des Aufwachsens und Jugendgestalten im 20. Jahrhundert (Frankfurt a.M. 1988) 11.

${ }_{7}$ Richard Kaufmann, Gebrannte Kinder. Die Jugend in der Nachkriegszeit (Düsseldorf 1961, TB-Ausgabe München 1966).

${ }^{8}$ Das wußte auch Helmut Schelsky, der sein Buch für das Verständnis der Gegenwartsjugend Mitte der 1960er Jahre ausdrücklich zurückzog. Ders., Zur Diskussion der Jugendsoziologie, Stellungnahme zu einem Buch und zu einer Rezension [von Leopold Rosenmayr, in: Kölner Zeitschrift f. Soziologie u. Sozialpsychologie 17 (1965) $182 \mathrm{ff}$.], in: ebd. 17 (1965) $401 \mathrm{ff}$.

9 Blücher, Generation, 12.

10 Ebd., $12 f$. 
allem Neuen gegenüber... Die Haltung ist - Gelassenheit. "11 Die „Generation der Unbefangenen" bilde eine "Jugend zunehmender, Normalisierung" und neuer Stabilisierung nach Jahrzehnten der Erschütterung. " ${ }^{12}$ Darin ähnele sie der Lebenswelt der Erwachsenen so sehr, daß Blücher für dieses Generationsverhalten der „Unbefangenen “ eine eigene Jugend- ${ }_{n}$ Subkultur “ glatt in Abrede stellt: „Eine jugendliche Subkultur findet in Deutschland nicht statt. ${ }^{~}{ }^{13}$ Ein Satz, zwei Jahre vor den Ereignissen von 1967/68 geschrieben, Ereignisse, die allerdings die gesamte damalige Jugendsoziologie in tiefe Ratlosigkeit versetzten. Dieser Sachverhalt mag aber auch als Hinweis darauf dienen, daß Blücher $z$ um einen keine "Generation“ (in einem unten noch zu explizierenden Sinne) beschrieben hat, sondern - wie er selber sagt - Mehrheiten und Minderheiten, aktive Gruppen und passive Zeitgenossen, und daß er zum anderen wohl eher die Oberflächenphänomene einer Generationslagerung registriert hat ( ${ }_{n}$ Jugend in ihrer Zeit $\left.{ }^{4}\right)^{14}$, die aber doch recht genau: die Bindungen junger Leute an Kirche und Familie seien ausgeprägt, aber eben pragmatisch; sie ließen sich nicht für weltanschauliche und ideologische Bindungen mißbrauchen; sie achteten die Demokratie und akzeptierten den Wehrdienst, aber Loyalität sei nicht mehr bedingungslos; das politische Interesse sei sehr ausgeprägt (die höchsten Werte aller Nachkriegsuntersuchungen!), politisches Engagement hingegen nur begrenzt; sie nutzten ibre Möglichkeiten in einer rasch sich wandelnden Gesellschaft. „Sie bleiben sich treu als die ,Unbefangenen'. "15 „Die Weltoffenheit, Aufgeschlossenheit, Vielseitigkeit, Wachheit, Interessenverzweigung, Funktionsfähigkeit und Handlungsbereitschaft der Generation der Unbefangenen lassen hoffen, daß sie dieser Aufgabe [der Entfaltung und Vervollkommnung des Gesellschaftssystems] gerecht werden können. ${ }^{~}{ }^{16}$

Zeitgleich veröffentlichte Elisabeth Pfeil ihre Paralleluntersuchung „Die 23jährigen ${ }^{17}{ }^{17}$ Einleitend wird das Konzept der "Generation" im Anschluß an Dilthey und Mannheim diskutiert ${ }^{18}$, sodann seine Transformation in den KohortenAnsatz der empirischen Sozialforschung im Anschluß an Ryder und Whelpton ${ }^{19}$.

11 Blücher, Generation, 14.

12 Ebd., 392.

13 Ebd., 396.

14 So auch Elisabeth Pfeil, Die 23jährigen. Eine Generationenuntersuchung am Geburtenjahrgang 1941 (Tübingen 1968); im folgenden zitiert: Pfeil, Die 23jährigen. - Zur konzeptionellen und begrifflichen Differenzierung von Generationslagerung, -zusammenhang und -einheit vgl. unten Abschn. II, 1.

15 Ebd., 402.

16 Ebd., 403.

17 Die Untersuchung mit 800 standardisierten und 200 Intensivinterviews wurde 1964-1967 durchgeführt. S. $12 \mathrm{ff}$. über die Vorgehensweise, $15 \mathrm{ff}$. über die statistischen Merkmale der Geburtenkohorte 1941.

$18 \mathrm{Vgl}$. unten Abschn. II, 1.

${ }^{19}$ Norman B. Ryder, The Cohort as a Concept in the Study of Social Change, in: American Sociological Review 30 (1965) 843-861, mit der Empfehlung, „generation“ should be used solely in its original and unambiguous meaning as the temporal unit of kinship structure* (853); im folgenden zitiert: Ryder, Cohort. - Vgl. auch Elisabeth Pfeil, Der Kohortenansatz in der Soziologie. Ein Zugang zum Generationsproblem?, in: Kölner Zeitschrift f. Soziologie 
Die Absicht der Untersuchung war, „Generations- ${ }^{\text {“ }}$ und Entwicklungsdaten miteinander zu verbinden, „um ihr jeweiliges Gewicht für das Generationsprofil zu ermessen" ${ }^{20}$. Den im Jahre 1964 23jährigen wird ein „profiliertes Generationsschicksal ${ }^{41}$ zugeschrieben: ,in der Kindheit Bombenangriffe, Evakuierung, Vertreibung, Abwesenheit des Vaters in Krieg und Gefangenschaft, Entnazifizierung des Vaters, Unterkunft in Notquartieren, also eine vielfache Belastung des Sozialisierungsvorganges in der frühen Kindheit, dann aber ein Hineinwachsen in die Berufswelt unter dem Zeichen wirtschaftlicher Expansion (mit Vollbeschäfrigung, ja Arbeitskräftemangel). ${ }^{22}$ In der Zusammenfassung der Befunde und der konzeptionellen Weiterführung ihrer Studie schreiben Elisabeth Pfeil und Jürgen Friedrichs ${ }^{23}$ - ähnlich wie Blücher - von einer entschiedenen "Lebenszuversicht" trotz der mannigfachen Behinderungen und Entbehrungen in der Kindheit ${ }^{24}$ und von einer ausgeprägten Aufstiegs- und Erfolgsorientierung; das „Grunderlebnis der fortschreitenden Stabilisierung " 25 ist der analoge Befund zu dem von Blücher.

Aber wie steht es mit der "Generation" der "68er", die in den Jahrgangskohorten dieser Befragungsgruppe ja auch enthalten sind ${ }^{26}$ ? Die Beantwortung dieser Frage führt auf die andere Frage, ob diese „Unbefangenen“ der Geburtsjahrgänge seit 1938/9 überhaupt eine "Generation" darstellen?

\section{Die Jahrgänge der „Unbefangenen“ 1939-1948: eine „Generation“?27}

Diese Frage ist schon zeitgenössisch aufgeworfen worden. Hans-Heinrich $\mathrm{Mu}$ chow, in der Denktradition von Eduard Sprangers „Psychologie des Jugend-

u. Sozialpsychologie 17 (1967) 645-657. - Der Ausdruck „Kohortenanalyse“ geht zurück auf: Pascal K. Whelpton, Cohort Analysis of Fertility, in: American Sociological Review 14 (1949) 735-749; ders., Cohort Fertility (Princeton 1954).

20 Pfeil, Die 23jährigen, 10.

21 Ebd., 8.

22 Ebd, 8. - Vgl. ähnlich unten Abschn. II, 3. - Pfeil, Die 23jährigen, charakterisiert, wie unten gezeigt wird, nicht ${ }_{n}$ die ${ }^{\text {"Generation }}$ " der 1941 Geborenen und zum Zeitpunkt der Untersuchung 23 jährigen, sondern eine Generationslagerung sowie eine mögliche Generationseinheit, die durch bestimmte Ereignisse und Erfahrungen geprägt worden war. Das muß sie aber nicht norwendigerweise von den Befunden, die sich auf die Generationslagerung beziehen, unterscheiden (vgl. unten Abschn. II, 4).

23 Pfeil, Die 23jährigen, dort: Pfeil, Das Generationsprofil der 23jährigen. Ihr Lebensgefühl und ihre Vorstellung vom gereiften Menschen, $349 \mathrm{ff}$.; Friedrichs, Theoretische Konsequenzen: Generationsproblem und Subkultur-These, $367 \mathrm{ff}$.

24 Ebd., 350.

25 Ebd., 353.

${ }^{26}$ In der Emnid-Gesamtgruppe waren nur 2\% Studenten. Da waren die wenigen entschieden Politisierten kaum auszumachen.

27 Die ausgedehnte Literatur zu den Themen "Generationskonzept " und "Generation und Jugend“ soll hier exemplarisch gesichtet werden, um damit zugleich die Beiträge und Diskussionen, die im vorliegenden Band dokumentiert werden, in die bisherige Forschungs- 
alters ${ }^{\text {28 }}$ an "Gestalten “ des Jugendlebens interessiert, konstatierte schon 1959

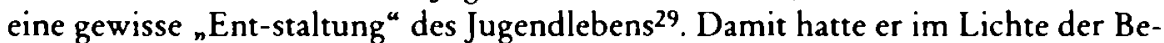
funde von Blücher gar keine so falsche Beobachtung gemacht. Aber was ist, wenn "die $e^{*}$ Jugend keine umrissene Gestalt als "Generation“ mehr erkennen läßt? Dann steht sie - in der Formulierung von Muchow und jedenfalls in seiner Wahrnehmung - „ungenau in dieser Welt" ${ }^{*}$.

In der Literatur herrscht Einigkeit darüber, daß es in einem „objektiven “ Sinne ${ }_{n}$ die $^{\alpha}$ Jugend weder als lebensgeschichtlich abgrenzbare Altersgruppe noch als homogene Lebensform gibt, nicht geben kann, sondern lediglich differierende Wahrnehmungs- und (Selbst-) Darstellungsformen von Gruppen und anderen formellen und informellen Gruppierungen, von unterschiedlichen Formen von Jugendkulturen und Jugendleben, von Fremd- und Selbstzuschreibungen zu - in Abgrenzung von anderen - gemeinsamen bzw. als gemeinsam empfundenen Lebensformen und Lebensstilen, Überzeugungen und Haltungen, Optionen, Praxen und Perspektiven. Die jeweilige Fokussierung durch den Betrachter - Altersgruppen und Lebenslagen, Fremdwahrnehmung und Selbstäußerung, Zugehörigkeit zu Geschlecht, Milieu und sozialer Schicht usw. - ergibt entsprechende „Bilder von Jugend bzw. Jugendleben. Das heißt: Die "Generation“ der „Unbefangenen“ bei Blücher ist keine Selbstzuschreibung oder Selbstwahrnehmung, sondern die empirisch mehr oder weniger gut begründete - Konstruktion von Gemeinsamkeiten der Angehörigen mehrerer, nicht beliebig zusammengestellter Jahrgänge (einer Kohorte), ohne daß damit behauptet würde, daß diese (oben zitierten) Gemeinsamkeiten erstens auf alle Angehörigen dieser Altersgruppe zuträfen (obwohl Blücher eine statistische Basis für Verallgemeinerungen durchaus ins Feld führen kann ${ }^{31}$ ), zweitens von allen Angehörigen in dieser Weise empfunden oder geteilt oder bewertet würden, drittens ausschließlich auf die Angehörigen dieser Jahrgänge zuträfen.

diskussion einzubetten. Vgl. dazu auch die vorzüglichen Analysen von Petersen, Radikale Jugend; dort die Kapitel II: Generationentheorie und Jugendtheorie, $39 \mathrm{ff}$., und VIII: Überprüfung und Modifikation der Generationentheorie anhand der jugendlichen Schweizer Generationsgestalten der Jahre 1900-1921, $515 \mathrm{ff}$.

${ }^{28}$ Eduard Spranger, Psychologie des Jugendalters (zuerst Leipzig 1924, zuletzt Heidelberg 291979). Zum Umfeld dieses überaus einflußreichen und für das Verständnis „des“ Jugendalters folgenreichen Buches vgl. Johannes-Christoph von Bühler, Die gesellschaftliche Konstruktion des Jugendalters. Zur Entstehung der Jugendforschung am Beginn des 20. Jahrhunderts (Weinheim 1990); Peter Dudek, Jugend als Objekt der Wissenschaften. Geschichte der Jugendforschung in Deutschland und Österreich 1890-1933 (Opladen 1990).

29 Hans-Heinrich Muchow, Sexualreife und Sozialstruktur der Jugend (Hamburg 1959).

30 Ebd., 126, 146.

31 Die "soziologisch-wirtschaftliche Struktur" seiner Stichprobe gleicht fast genau der amtlichen Statistik hinsichtlich Geschlecht und Länderverteilung sowie nach Bildungsklassen und Altersgruppen der Bevölkerungsstatistik des Jahres 1961; das Befragungsergebnis läßt sich mithin auf diese Grundgesamtheit "Gesamtbevölkerung" verallgemeinern (Blücher, Generation, 15, Daten 404 f.). 
Für die weitere Erörterung ist es daher unabdingbar, einige sprachlich-begriffliche und konzeptionell-theoretische Klärungen vorzunehmen, um eine Verständigung darüber vorzuschlagen, was unter "Generation" verstanden werden soll: z. B. als kollektive Akteure in geschichtlichen Handlungszusammenhängen (z. B. die jugendbewegten bzw. freideutschen Kriegsfreiwilligen 191432, aktive Gruppen z. B. in einer Partei ${ }^{33}$ oder in einer Epoche ${ }^{34}$, die ${ }_{n} 45 \mathrm{er}^{-}{ }^{\circ}$ im Vergleich mit der "68er" ${ }_{n}$ Generation $^{\star 35}$, in der Wissenschaftsgeschichte ${ }^{36}$ ), in geschichts- und so-

$32 \mathrm{Vgl}$. als instruktives Beispiel Gudrun Fiedler, Jugend im Krieg. Bürgerliche Jugendbewegung, Erster Weltkrieg und Sozialer Wandel 1914-1923 (Edition Archiv der deutschen Jugendbewegung, Köln 1989); Dietmar Schenk, Die Freideutsche Jugend 1913-1919/20. Eine Jugendbewegung in Krieg, Revolution und Krise (Geschichte der Jugend, 17, Münster i.W. 2000).

33 Detlef Lehnert, Sozialdemokratie zwischen Protestbewegung und Regierungspartei 18481983 (Frankfurt a.M. 1983) 10: vermittelt werden, dic Geschichte der Sozialdemokratie als sozialen und politischen Lernprozeß, d.h. aus epochenspezifischen Erfahrungen ihrer Funktionsträger und Mitglieder verstchen zu können. Da ein solches Modell programmatischer und praktischer Lernschritte die konkrete Auseinandersetzung mit staatlichen und gesellschaftlichen Herrschaftsverhältnissen im Bewußtsein und Handeln von Sozialdemokraten unterstellt, wird als Bezugspunkt der Verarbeitung von Erfahrungen jeweils eine "Parteigeneration" angenommen [sic!]. Thematisch einschlägig Günter Bannas, Wen lassen die Enkel an die Fleischtöpfe? Der sozialdemokratische Generationenkonflikt, in: Frankfurter Allgemeine Zeitung Nr. 134 vom 13.6. 2002, 3; die "Kopfleiste" der Süddeutschen Zeitung Nr. 168 vom 23. 7.2002 lautete: „Hundestag: Die Generation Kohl verabschiedet sich“ (Verzicht aus Altersgründen auf erneute Kandidaturen).

34 Martin Doerry, Übergangsmenschen. Die Mentalität der Wilhelminer und die Krise des Kaiserreichs (Weinheim, München 1986) 30 ff.: Auf der Suche nach einer Generation; Christian Schneider, Cordelia Stillke, Bernd Leineweber, Das Erbe der Napola. Versuch einer Generationengeschichte des Nationalsozialismus (Hamburg 21997); hier wird übrigens nicht "Generationen"-Geschichte beschrieben, sondern die Geschichte von Vätern und Söhnen. Von "Generation “ ist mithin gar nicht i.S. der Terminologie einer Historik die Rede, sondern - wie auch sonst öfters in neuerer Literatur (vgl. Anm. 43 ff.) - in einem nicht-terminologischen Alltagsverständnis der Umgangssprache.

$35 \mathrm{Vgl}$. den anregenden Essay von Günter Gaus, Wer den Mund zu voll nimmt. Die 45er und die 68er verbindet nur eins: Beide Generationen beanspruchen das Recht auf eigene Irrtümer, in: Süddeutsche Zeitung Nr. 17 vom 9. 3.2001, 17.

36 Hier handelt es sich häufig um "Schulen" bzw. "Arbeitsgruppen“, die insbesondere durch Neuentwicklungen ("Paradigmenwechsel ${ }^{*}$ ) auf sich aufmerksam machen. Vgl. Rüdiger vom Bruch, Brigitte Kaderas (Hrsg.), Wissenschaften und Wissenschaftspolitik. Bestandaufnahme zu Formationen [sic!], Brüchen und Kontinuitäten im Deutschland des 20. Jahrhunderts (Stuttgart 2002). Der Versuch einer „Generationengeschichte der Kritischen Theorie“ - so der Untertitel des Buches "Trauma und Kritik" von Christian Schneider, Cordelia Stillke, Bernd Leineweber (Münster 2000) - kann mit einem analytischen Generationsbegriff nichts anfangen und verkennt daher (33ff.) die Leistungsfähigkeit und die Anforderungen an eine Generationsgeschichte. Die Ausführungen dort über „Kritische Theorie im Generationenverhältnis" (131 ff.) handeln konventionell vom Lehrer-Schüler-Verhältnis anhand der Differenzen und Distanzierung von Habermas und Adorno. - Diese Position wurde auch zusammenfassend vorgetragen von Christian Schneider, Noch einmal "Geschichte und Psychologie $^{\prime}$. Generationengeschichte am Modell psychohistorischer Forschung, in: Mittelweg 36, Bd. 6 (1997) H. 2: 83-92; H. 3: 45-56. Unter Psychohistorie versteht Schneider übrigens eine psychologische bzw. psychoanalytische Interpretation von wissenschaftlichen Kontroversen 
zialwissenschaftlichen Erklärungszusammenhängen (z.B. die Genese der politischen Optionen und des politischen Potentials politischer Bewegungen ${ }^{37}$, von Protestbewegungen der Jugend, vor allem der "68er" ${ }^{48}$ ), in Deutungszusammenhängen Sozial-kulturellen Wandels: etwa zur politischen Kultur sowie zu Generationenbeziehungen, -spannungen, -konflikten ${ }^{39}$ und -segregationen ${ }^{40}$, zur sozialen Dynamik in intergenerationellen Ablösungsprozessen ${ }^{41}$ sowie inzwischen sogar zum Thema "Globalisierung ${ }^{42}$. - Unberücksichtigt bleiben Abhandlungen

und Differenzen und nicht, wie in der Geschichtswissenschaft üblich, entweder die Geschichte von Mentalitäten, Einstellungen usw. oder die Historizität der Strukturen und Bedeutungen des Psychischen.

37 Zum Beispiel Michael H. Kater, Generationskonflikt als Entwicklungsfaktor in der NS-Bewegung vor 1933, in: Geschichte und Gesellschaft 11 (1985) 217-243; Jürgen W. Falter, Die Jungmitglieder der NSDAP zwischen 1925 und 1933. Ein demographisches und soziales Profil, in: Wolfgang A. Krabbe (Hrsg.), Politische Jugend in der Weimarer Republik (Dortmunder Historische Studien 7, Bochum 1993) 202-221; Andreas Petersen, Radikale Jugend. Die sozialistische Jugendbewegung der Schweiz 1900-1930. Radikalisierungsanalyse und Generationentheorie (Zürich 2001); im folgenden zitiert: Petersen, Radikale Jugend.

38 Zur 68er- „Generation“ ist die Literatur inflationär. Grundlegend sind die Materialsammlungen, die von Wolfgang Kraushaar herausgegeben wurden: Die Protest-Chronik 19491959. Eine illustrierte Geschichte von Bewegung, Widerstand und Utopie (4 Bde., Hamburg 1996); Frankfurter Schule und Studentenbewegung. Von der Flaschenpost zum Molotowcocktail 1946-1996 (3 Bde., Hamburg 1998).

39 Theodor Litt, Das Verhältnis der Generationen ehedem und heute (Wiesbaden 1947); Manfred Riedel, Wandel des Generationenproblems in der modernen Gesellschaft (Düsseldorf, Köln 1969); der vorzügliche Band von Dieter Dowe (Hrsg.), Jugendprotest und Generationenkonflikt in Europa im 20. Jahrhundert. Deutschland, England, Frankreich und Italien im Vergleich (Bonn 1986).

$40 \mathrm{Vgl}$. M. Kent Jennings, Klaus Allerbeck, Leopold Rosenmayr, Generations and Families, in: Samuel H. Barnes et al. (Hrsg.), Politicial Action. Mass Participation in Five Western Democracies (Beverly Hills, London 1979) 449-522; Walter Jaide, Generationen eines Jahrhunderts. Wechsel der Jugendgenerationen im Jahrhunderttrend. Zur Sozialgeschichte der Jugend in Deutschland 1871-1985 (Opladen 1988); im folgenden zitiert: Jaide, Generationen; Fend (wie Anm. 6). - Es gibt auch den Beschreibungsversuch von Heinz Bude, Das Altern einer Generation. Die Jahrgänge 1938-1948 (Frankfurt a.M. 1995), nur handelt es sich bei dem willkürlichen Ensemble von einigen Repräsentanten dieser Jahrgänge, wie unten zu zeigen sein wird, um keine "Generation", und das Kapitel "Das Entstehen einer Generation“ ( $37 \mathrm{ff}$.) handelt von allem möglichen, aber nicht davon.

${ }^{41}$ Dazu immer noch als jugendsoziologischer Klassiker Shmuel N. Eisenstadt, Von Generation zu Generation. Altersgruppen und Sozialstruktur (zuerst 1956, München 1966). Zu sozialstrukturellen Aspekten vgl. Rolf Becker (Hrsg.), Generationen und sozialer Wandel. Generationsdynamik, Generationsbeziehungen und Differenzierung von Generationen (Opladen 1997)

42 Zum Beispiel die 68er als einer „weltweiten Generationselite“: Beate Fietze, 1968 als Symbol der ersten globalen Generation, in: Berliner Journal für Soziologie 7 (1997) 365-387. Der Epocheneinschnitt von 1989 in der (nicht nur) deutschen Geschichte wird von Claus Leggewie, Die 89er. Porträt einer Generation (Hamburg 1995) zum Ausgangspunkt genommen, um eine neue Generation (der 13- bis 30jährigen) zu beschreiben, die die „Berliner Republik“ maßgeblich bestimmen wird. Vgl. auch: ders., „Ihr kommt nicht mit bei unseren Änderungen!“ Die 89er - Generation ohne Eigenschaften?, in: Transit. Europäische Revue 11 (1996) 3-17. Beide Texte von Leggewie sind keine empirischen soziologischen bzw. zeitgeschichtlichen Analysen, sondern anregende Essays für das Verstehen zeitgeschichtlicher 
und Untersuchungen, die von „Generation" lediglich im nicht-terminologischen und im nicht historisch-analytischen Sinn sprechen: als pädagogisches Verhältnis von Eltern und Kindern i.S. des Erfahrungsabstandes der Älteren von und ihrer Verantwortung für die Jüngeren ${ }^{43}$; unter dem Aspekt des Zusammenlebens von Älteren und Jüngeren ${ }^{44}$ und dabei auch unter sozialpsychologischen Aspekten ${ }^{45}$; njunge Generation " als Lebensalter ( ${ }^{\text {Jugend }}{ }^{\text {") }}$ und als Sozialisationsphase ("Jugendalter $\left.{ }^{4}\right)^{46}$; das Konzept der Generation als Altersabstand und seine Ausdifferenzierung in der Historischen Familienforschung wird hier ebenfalls ausgeblendet ${ }^{+7}$.

\section{1) Das Konzept der "Generation"}

"Generations“-Zugehörigkeit wurde in der deutschen Geistes- und Sozialgeschichte von zwei bedeutenden Forschern bei der Beschäftigung mit zwei konträren Phänomenen von "Generations"-Zugehörigkeit thematisiert: zuerst von

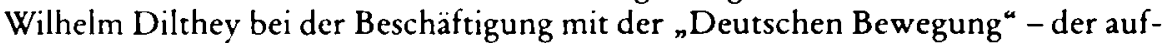
fälligen Gleichzeitigkeit der Dichter der Klassik und Romantik - und dann von Karl Mannheim bei der Analyse der Differenzen von Älteren und njunger Generation " in den 1920er Jahren.

Prozesse und Herausforderungen, bevor die Eule der Minerva ihren Flug beginnen kann. Im übrigen können ndie“ 13- bis 30jährigen keine "Generation" sein oder bilden.

${ }^{43}$ Diese pädagogische Denkform geht auf Schleiermachers Pädagogik (1826) zurück. Vgl. Jutta Ecarius (Hrsg.), Was will die jüngere mit der älteren Generation? Generationenbeziehungen in der Erziehungswissenschaft (Opladen 1998). Der Haupttitel dieses Buches ist eine Umkehrung der Schleiermacherschen Frage "Was will die ältere Generation mit der jüngeren?" als der systematischen Ziel- und Zweckfrage der Pädagogik. Diese Umkehrung findet sich bei Burkhard K. Müller in: Eckart Liebau, Christoph Wulf (Hrsg.), Generationen. Versuche über cine pädagogisch-anthropologische Grundbedingung (Weinheim, München 1996) $304 \mathrm{ff}$.; in beiden Bänden auch Abhandlungen zu Schleiermacher. - Die pädagogische Diskussion resümierend, ansonsten ohne weiterführenden Belang (wie der Untertitel schon andeutet) Hans-Rüdiger Müller; Das Generationenverhältnis. Uberlegungen zu einem Grundbegriff der Erziehungswissenschaft, in: Zeitschrift für Pädagogik 45 (1999) 787-805. ${ }^{44}$ Micha Brumlik, Gerechtigkeit zwischen den Generationen (Berlin 1995); Eckart Liebau (Hrsg.), Das Generationenverhältnis. Über das Zusammenleben in Familie und Gesellschaft (Weinheim, München 1997).

45 Helga Margarate Merker, Generations-Gegensätze. Eine empirische Erkundungsstudie über die Einstellung Erwachsener zur Jugend (Darmstadt 1973); Ame Stikstud, Jugend im Generationen-Kontext. Sozial- und entwicklungspsychologische Perspektiven (Opladen 1994), bes. 157 ff. zu Generations-Konflikten und - Wahrnehmungen.

46 Friedbelm Neidhardt, Die junge Generation. Jugend und Gesellschaft in der Bundesrepublik (Opladen ${ }^{3} 1970$ ), zugleich in: Deutsche Gesellschaft im Wandel (Bd. 2, Opladen 1970) 85-186; vgl. auch Ulf Preuß-Lausitz u.a., Kriegskinder, Konsumkinder, Krisenkinder. Zur Sozialisationsgeschichte seit dem Zweiten Weltkrieg (Weinheim, Basel ${ }^{21989}$ ), wo eine "Sozialisationsgeschichte in Generationen" (S. 11) vorgeschlagen, aber leider nicht wirklich eingelöst wird.

47 Vgl. die anregende Skizze von Thomas Schuler, Der Generationsbegriff und die Historische Familienforschung, in: Peter-Johannes Schuler (Hrsg.), Die Familie als sozialer und historischer Verband (Sigmaringen 1987) 23-41. 
Den Ausgangspunkt des modernen "Generationen"-Konzepts bilden Überlegungen Wilhelm Diltheys. Von seinem Novalis-Essay von $1865^{48}$ über seine Basler Antrittsvorlesung im Jahre 1867 „Die dichterische und philosophische Bewegung in Deutschland 1770-1800449 und weiter zu seiner für die Theoric der modernen Geisteswissenschaften grundlegenden Abhandlung von 1875 "Über das Studium der Geschichte der Wissenschaften vom Menschen, der Gesellschaft und dem Staat ${ }^{\alpha 5}$ - der frühesten Konzeption des Programms einer „Kritik der historischen Vernunft" in seiner "Einleitung in die Geisteswissenschaften " von 1883 - bis zu zentralen Passagen in seinem Spätwerk „Der Aufbau der geschichtlichen Welt in den Geisteswissenschaften " 51 findet sich immer wieder das Konzept der „Generation“; denn Dilthey war auf der Suche nach analytischen Begrif-

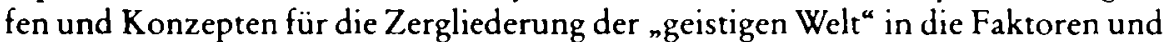
Strukturen ihrer „Wirkungszusammenhänge“, um ein „Gerüst des Verlaufs geistiger Bewegungen " zu gewinnen (das meint "Kritik der historischen Vernunft ${ }^{*}$ ): das einzelne Menschenleben, die Lebensalter, die Generation und von dort weiter zu einer Strukturgeschichte von "Systemen der Kultur" und "Systemen der äußeren Organisation ${ }^{4}$ der Gesellschaft ${ }^{52}$.

Die Abhandlung von 1875 enthält die konzeptionell präzise Formulierung: "Generation ist ... eine Bezeichnung für ein Verbältnis der Gleichzeitigkeit von Individuen; diejenigen, welche gewissermaßen nebeneinander emporwuchsen, das heißt ein gemeinsames Kindesalter hatten, ein gemeinsames Jünglingsalter, deren Zeitraum männlicher Kraft teilweise zusammenfiel, bezeichnen wir als dieselbe Generation. Hieraus ergibt sich dann die Verknüpfung solcher Personen durch ein tieferes Verhältnis. Diejenigen, welche in den Jahren ihrer Empfänglichkeit dieselben leitenden Einwirkungen erfahren, machen zusammen eine Generation aus. So gefaßt, bildet eine Generation einen engeren Kreis von Individuen, welche durch Abhängigkeit von denselben großen Tatsachen und Veränderungen, wie sie in dem Zeitalter ihrer Empfänglichkeit auftraten, trotz der Verschiedenheit hinzutretender anderer Faktoren zu einem homogenen Ganzen verbunden sind. ${ }^{\text {53 }}$

48 Novalis, zuerst 1865, wiederabgedr. in: ders., Das Erlebnis und die Dichtung (Leipzig 1906, zit. Göttingen $\left.{ }^{14} 1965\right)$ 187-241.

49 Aus dem Nachlaß zuerst in: ders., Gesammelte Schriften (Bd. V, Leipzig 1924, zit. Stuttgart, Göttingen 41964) 12-27.

50 Zuerst 1875, wiederabgedr. in: ders., Gesammelte Schriften (Bd. V, Leipzig 1924, zit. Stuttgart, Göttingen $\left.{ }^{4} 1964\right)$ 31-73.

51 Berliner Akademie-Abhandlungen der Jahre $1905 \mathrm{ff}$, jetzt in: ders., Gesammelte Schriften (Bd. VII, Leipzig 1927, zit. Stuttgart, Göttingen 21958 u.ö.).

52 Das wird dann in der "Einleitung in die Geisteswissenschaften" (zuerst 1883, jetzt in: ders., Gesammelte Schriften, Bd. I, zuerst Leipzig 1883, dann in der Werkausgabe Leipzig 1922, ab 4. Aufl. Stuttgart, Göttingen 1959 u.ö.) begründet und in den Studien zum „Aufbau der geschichtlichen Welt in den Geisteswissenschaften " (wie Anm. 51) weiter differenziert. Dort verweist Dilthey in einem Abschnitt "Zeitalter und Epochen" (S. $177 \mathrm{ff.}$.) - wiederum mit Verweis auf seine Novalis-Abhandlung - im Hinblick auf geschichtliche Kontinuität, Bewegung und Epochenspezifik auf die zentralen Begriffe "Generation", "Zeitalter" und „Epoche" (S. 177, Anm. 1).

$\$ 3$ Wie Anm. 50, 35, Hervorhebung von WD. Unmittelbar anschließend zitiert Dilthey dann 
Dilthey hat freilich selber auf die Grenzen des methodischen Kunstgriffs hingewiesen, "Generationen" zu "konstruieren", wenn sie selber nicht als solche offenkundig sind bzw. sich selber nicht als solche verstehen: „Die Form der historischen Darstellung täuscht so leicht über dieselben [gemeint ist: die Grenzen der Operationen zur Bestimmung von "Generationen"]. Denn sie [die Form der historischen Darstellung] schreitet überall mit der Zeit selber vorwärts, ableitend, aus Ursachen Folgen entwickelnd ... Dies Verfahren ist nur ein schöner Schein (sic!) der Kunst des Geschichtsschreibers. " ${ }^{54}$ In seiner Novalis-Abhandlung hatte Dilthey formuliert, daß mit einem solchen Konzept und seiner Vorgehensweise die methodologische Grenze zu beachten sei: „Hierbei ist aber die wahre Natur (sic!) unseres Verfahrens mit den geschichtlichen Bedingungen hervorzuheben. Wir lassen nämlich den allergrößten Teil derselben (i.e. der , intellektuellen Kultur" und des "umgebenden Lebens") ganz außer Rechnung und behandeln eine begrenzte Reihe (sic!), die wir aus ihnen aussondern, ohne weiteres als Totalität (sic!) derselben. Wenn wir also den Anspruch machen, sie durch unsere Analyse darzustellen, so kann schon aus diesem Grunde der Anspruch nur auf eine sehr approximative (sic!) Richtigkeit gehen. Wir erklären nur aus den bervorragendsten Bedingungen. ${ }^{.55}$

Von Diltheys Überlegungen ist festzuhalten:

(1) das Bedingungsverhältnis der geistigen Aktivitäten des nheranwachsenden Geschlechts" und der strukturellen Bedingungen der Lebensumstände;

(2) "Generation" ist ein gemeinsames lebensgeschichtliches „Verhältnis der Gleichzeitigkeit von Individuen“, wodurch sie sich von anderen Zeitgenossen unterscheiden (lassen);

(3) eine solche "Generation", wenn sie nicht selber als solche in Erscheinung tritt, ist eine Konstruktion des Historikers: er blendet diese "Generation" als eine „begrenzte Reihe“ aus der Gesamtheit der Zeitgenossen bzw. ihrer Kohorten aus.

Damit hat Dilthey "Generation" als analytisches Instrument und als "Denkform " einer intellectual history folgenreich bestimmt: Es dient ihm dazu, das zeitgenössisch gleichzeitig Andere sichtbar zu machen, was dann später (bei Karl Mannheim) Generationseinheit innerhalb einer Generationslagerung und eines Generationszusammenhangs (innerhalb der Lagerung) genannt wurde. Was Wilhelm Dilthey als systematisch orientierter Theoretiker der Geistes- und Kulturgeschichte als analytisch-begriffliche Kategorie stringent zu entwickeln und begründen suchte und andererseits zugleich als auf Differenzierung bedachter $\mathrm{Ge}$ schichtsschreiber als Erkenntnisform relativierte und überdies unter den Vorbehalt der Perspektivität der Geschichtlichkeit stellte - im Lichte der sich auf Dilthey beziehenden Literatur übrigens recht erfolgreich ${ }^{56}$-, wurde von dem Soziologen

die hier zuvor erwähnte Passage aus der Novalis-Abhandlung, auf die er selber anmerkungsweise hinweist.

54 Ebd., 38.

55 Wie Anm. 48, 188.

56 Vor allem folgende Schriften werden immer wieder aufgeführ: Julius Petersen, Die Wesensbestimmung der deutschen Romantik. Eine Einführung in die moderne Literaturwis- 
Karl Mannheim am Ende der 20er Jahre in einer ausufernden Debatte über „Generationen " nicht minder erfolgreich als soziologisches Ordnungsinstrument eingeführt ${ }^{57}$. Mannheim unterscheidet die Generationslagerung im konkreten räumlich-zeitgeschichtlichen Lebensraum, darin den Generationszusammenhang derjenigen annähernd Gleichaltrigen, die ein gemeinsamer Erfahrungshorizont verbindet, innerhalb dessen sich Generationseinheiten als Erlebnisgemeinschaften herauskristallisieren, die sich historisch als politisch, kulturell, gesellschaftlich aktive Gruppen - kollektive Akteure - bemerkbar machen. Breitsamer hat eine interessante Studie vorgelegt, in der er Mannheims Analyse durch quantitative und qualitative Daten unterlegt - hier: kategoriale Ereignisse 1918, 1933, 1945; Wirtschaftskrisen; politische Optionen bei Wahlen - und bestätigt ${ }^{58}$. Fogt nimmt dieses Konzept als Ausgangspunkt für seinen weiterführenden Versuch, die (Selbst-)Konstitution von politisch aktiven Generationseinheiten wie die "68er" zu erklären ${ }^{59}$. Petersen ${ }^{60}$ meint, daß ihm das nicht ganz schlüssig gelungen sei, knüpft seinerseits ebenfalls an Mannheim an und fügt dessen Ansatz bedenkenswerte Differenzierungen hinsichtlich der Formierungsprozesse von Generationseinheiten hinzu ${ }^{61}$.

Mit dem Aufstieg der empirischen Sozialforschung mußte die Kategorie "Generation " einer ${ }_{n}$ verstehenden (Jugend-)Soziologie " problematisch werden; denn sie eignet sich offensichtlich nicht als quantifzierendes Ordnungsinstrument im Rahmen einer empirischen sozialwissenschaftlichen Jugendforschung, die sich für aktuelle Einstellungen und Verhaltensweisen von Jahrgängen bzw. Jahrgangs-Kohorten interessierte, sei es im Bereich der Wahlforschung und (Jugend-)Politikberatung, sei es im Hinblick auf größere Zusammenhänge des Sozialen Wandels. Die Diskussion begann früh in den USA 62 im Zusammenhang mit der Erforschung des Verhältnisses von Altersgruppen zueinander. In diesem Zusammenhang mache

senschaft (Leipzig 1926, Reprint Heidelberg 1968), Kap. VI: Generation, 132-170; ders., Die literarischen Generationen (Berlin 1930); Wilhelm Pinder, Kunstgeschichte nach Generationen (Leipzig 1926); ders., Das Problem der Generation in der Kunstgeschichte Europas (Berlin 21928); Eduard Wechssler, Die Generation als Jugendreihe und ihr Kampf um die Denkform (Leipzig 1930).

57 Karl Mannbeim, Das Problem der Generationen, in: Kölner Zeitschrift für Soziologie 7 (1928) $157 \mathrm{ff}$., $309 \mathrm{ff}$, wiederabgedr. in: ders., Wissenssoziologie, hrsg. von $K$. H. Wolff (Soziol. Texte, Bd. 28, Neuwied, Berlin 21970) 509-565. - Zu Mannheim neuerdings Joachim Matthes, Karl Mannheims "Das Problem der Generationen“, neu gelesen. Generationen"Gruppen " oder „gesellschaftliche Regelung von Zeitlichkeit“", in: Zeitschrift für Soziologie 14 (1985) 363-372; Jane Pilcher, Mannheim's sociology of generations: an undervalued legacy, in: The British Journal of Sociology 45 (1994) 481-495; dies., Age and Generation in Modern Britain (Oxford 1995); vgl. den Beitrag von Jürgen Zinnecker (Siegen) in diesem Band.

58 Joachim Breitsamer, Ein Versuch zum „Problem der Generationen“, in: Kölner Zeitschrift f. Soziologie u. Sozialpsychologie 28 (1976) 451-478.

59 Helmut Fogt, Politische Generationen. Empirische Bedeutung und theoretisches Modell (Beiträge zur sozialwiss. Forschung, 32, Opladen 1982).

60 Petersen, Radikale Jugend.

61 Ebd., $529 \mathrm{ff}$.

62 Ryder, Cohort. 
die Rede von „Generation“ keinen Sinn - was ganz richtig beobachtet ist -, denn in Gesellschaften mit zunehmender Dynamik des Sozialen Wandels sei die Milieuund die Kobortenzugehörigkeit wichtiger als die (wie auch immer definierte) Generationenzugehörigkeit ${ }^{63}$. Ähnlich wurde auch 30 Jahre später im Zusammenhang der Transformation der beschreibenden und verstehenden Jugendsoziologie (vom Schlage Schelskys oder Blüchers) in eine empirische und erklärende Jugendforschung argumentiert ${ }^{64}$. Erfolgreiche Kohortenanalysen ${ }^{65}$ führten einerseits zu der Empfehlung, den Begriff der Generation im Sprachgebrauch der Soziologie zu meiden ${ }^{66}$ - was nicht ohne Widerspruch blieb ${ }^{67}$-, andererseits aber auch zu dem interessanten Vorschlag, das Kohortenkonzept zu nutzen, um das Generationskonzept für die empirische Sozialforschung operationalisiert nutzbar zu machen ${ }^{68}$.

${ }^{63}$ Ebd., 853; Irving Rosow, What is a Cohort and why?, in: Human Developoment 212 (1978) 65-75 zitiert S. 66 Peter B. Baltes (den späteren Direktor am MPI für Bildungsforschung in Berlin) mit der Auffassung, daß aus der Sicht der Sozial- und Entwicklungspsychologie ,available evidence on the role of cohort effects on behavioral development is largely descriptive; efforts at theoretical-explanatory analysis arc rare and at the best prototheoretical“", so daß Kohortenanalysen den Historikern und Soziologen überlassen wurden. Rosow schlägt vor, daß cine Kohorte als im soziologischen Sinne "meaningful entity " ${ }_{n}(1)$ consists of people who share a given life experience; (2) this experience is socially or historically structured, and (3) it occurs in a common generational framework; (4) its effects distinguish one generation from another; and (5) these effects are relatively stable over the life course." (67) Damit ist eine pragmatische Definition gegeben, um nicht von vornherein vor dem eigentlichen Problem der Abgrenzung und Differenzierung von Kohorten(-Effekten) zu kapitulieren und die deshalb vor allem für Kohorten-Effekte sensibilisieren soll; in der geschichtlichen und gesellschaftlichen Realität indes „clear-cut cohort effects do not occur as often as we expect". (74)

64 Walter Hornstein, Christian Lüders, Das Problem der Generationen in der Jugendforschung heute. Anmerkungen aus pädagogischer Sicht, in: Bildung und Erziehung 38 (1985) 213-230, mit der Empfehlung, das Konzept der Generation durch das der „Lebenslage“ bzw. "Problemlage" zu ersetzen (226). (Dilthey hatte von "Lebenshorizont" gesprochen.) Das "Lebenslagen"-Konzept wird zuerst, soweit ich sehe, in der jugendsoziologischen Forschung von Ulrich Planck benutzt (vgl. Anm. 92). Der Empfehlung von Hornstein/Lüders ist die sozialwissenschaftliche und sozialpädagogische Jugendforschung sehr erfolgreich gefolgt.

$65 \mathrm{Vgl}$. Wolfgang Plum, Kohortenanalyse von Umfragedaten. Zur Identifizierung möglicher Einflußfaktoren politischen Verhaltens und politischer Einstellungen, in: Kölner Zeitschrift f. Soziologie u. Sozialpsychologie 34 (1982) 509-532.

66 Gerhard Schmied, Der soziologische Generationsbegriff. Darstellung, Kritik und „Gewissenserforschung “, in: Neue Sammlung 24 (1984) 231-244.

67 Vom Vf.: Das Konzept der "Generation“. Ein Forschungs- und Erklärungsansatz für die Erziehungs- und Bildungssoziologie und die Historische Bildungsforschung, in: Neue Sammlung 27 (1987) 364-377, wiederabgedr. in: ders. (Hrsg.), Jugendpolitik in der Nachkriegszeit (Weinheim, München 1993) 99-117; ders., Neue Wege der Sozialgeschichte. Zur Forschungspraxis der Historischen Sozialisationsforschung und zur Bedeutung ihrer Ergebnisse für pädagogische Theoriebildung, in: Pädagogische Rundschau 38 (1984) 171-187. 68 Bernd Buchbofer, Jürgen Friedrichs, Hartmut Lüdtke, Alter, Generationsdynamik und soziale Differenzierung. Zur Revision des Generationsbegriffs als analytisches Konzept, in: Kölner Zeitschrift f. Soziologie u. Sozialpsychologie 22 (1970) 300-334. 
Diese Debatte wurde von den zeitgeschichtlichen Ereignissen überholt: Seit Mitte der 60er Jahre war eine internationale Studentenbewegung als eine "neue Generation" wahrnehmbar, so wie Dilthey es von den Romantikern gesagt hatte, und Lewis S. Feuer gab seiner aktuell-zeitgenössischen, international vergleichenden voluminösen Studie den zutreffenden Titel "The Conflict of Generations. The Character and Significance of Student Movements "69. Damit war das Generationen-Thema wieder auf der Tagesordnung und ist es in der (Jugend-)Soziologie sowie der Geschichtswissenschaft ${ }^{70}$ auch geblieben.

Helmuth Plessner, an den hier erinnert werden muß, sollte Recht behalten. Er hatte in seinem bis heute überaus lesenswerten Essay zu unserem Thema geschrieben ${ }^{71}$, daß bei der Debatte über das Konzept der Generation der Diltheysche Ansatz und dessen eigentliches Erkenntnisinteresse zu wenig beachtet worden sei (übrigens auch von Karl Mannheim): Dilthey habe sich für Generationseinheiten interessiert, weil es ihm um das Eingrenzen und Verstehen geistiger Bewegungen gegangen sei, und zwar in der Weise, daß für ihn nicht die Abfolge, sondern die Gleichzeitigkeit dieser Bewegungen der erklärungsbedürftige Sachverhalt sei. Und Plessner fuhr fort: „Die Einheit der Generation erscheint ... als Gruppe dank der Anziehungskraft auf Menschen gleichen Alters, die sich dadurch, das heißt durch das, was sie damals geglaubt und mitgemacht, und wie sie es mitgemacht haben, einander verwandt fühlen. ... Ein solcher Generationsbegriff schließt enger an die soziale Wirklichkeit an als der scheinbar unübertreffliche Realismus der naturalistischen Theoretiker ${ }^{72}$. Der enge Anschluß an den soziokulturellen Aspekt bedeutet jedoch, daß die biologische Marke des Geburtsjahrganges ihre Bedeutung verliert. Situation wird wichtiger als Generation. Aber auch die Situation verliert an Schärfe der Begrenzung zugunsten der Bindekraft des zündenden Gedankens. Die Richtung wird das Bindemittel und erweist sich als die eigentliche sozialisierende Kraft. Das Bewußtsein eines gemeinschaftlichen Zieles verdrängt - im konkreten Fall - die Gemeinsamkeit durch gleiches Alter. Und in dem Maße, in welchem das Lebensalter von der Gemeinsamkeit der Lebenssituation und diese wiederum von der einer Überzeugung in den Schatten gestellt wird, bildet sich innerhalb bestimmter Gruppen eine ,Generation' im geschichtlich faßbaren Sinn heraus. Die dem gleichen Jahrgang angehörenden Führer einer Schule, einer Bewegung werden sich ihrer Generationslage nur bewußt werden, wenn sie ihr eine Bedeutung zuschreiben. Ohne ein derartiges Generationsbewußtsein gibt es also

69 New York, London 1969.

70 Als frühe Beispiele: Alan B. Spitzer, The Historical Problem of Generations, in: American Historical Review 78 (1973) 1353-1385; Hans Jäger, Generationen in der Geschichte. Überlegungen zu einer umstrittenen Konzeption, in: Geschichte und Gesellschaft 3 (1977) 429-452; im folgenden zitiert: Jäger, Generationen.

71 Nachwort zum Generationenproblem (1949/1966), in: ders., Gesammelte Schriften (Bd. X, Frankfurt a.M. 1985) 107-120.

72 Gemeint ist offenbar die Orientierung am "naturalistischen“ Basisdatum „Geburtsjahrgang". 
keine Generationsbildung mit dem vitalen Akzent, keine Gruppenbildung im Zeichen naturgegebener Momente. ${ }^{473}$

Nach diesem kurzen Durchgang durch die Geschichte des Generationen-Konzepts sei eine Zusammenfassung versucht, was im Unterschied zu Jahrgang und Kohorte unter „Generation " verstanden wird bzw. verstanden werden soll, wenn dieser Begriff für geschichtstheoretische bzw. historiographische Zwecke einigermaßen präzise handhabbar sein soll:

(1) Ein Jabrgang ist eine eindeutige zeitliche Zuordnung im Ablauf der Geschichte, die Jahrgangszugehörigkeit ist für jedermann durch sein Geburtsdatum eindeutig. Aus der Jahrgangszugehörigkeit ergeben sich oft überraschende Gemeinsamkeiten, wie sich z.B. an den Jahrgangs- "Spiegeln“ in der "Frankfurter Allgemeinen Zeitung “ seit 1980 für die Jahrgänge seit 1920 ablesen läßt ${ }^{74}$.

1920-1923 Kriegsjahrgänge, sehr dezimiert; guter Start in der Nachkriegszeit: die Überlebenden waren rechtzeitig zum Wiederaufbau wieder zur Stclle und wuchsen Schritt für Schritt in ihre Funktionen und Positionen hinein; hoher Zuzug aus der SBZ/DDR

1924 der erste "normale" Jahrgang in der Jahrgangsgeschichte der BRD

1925 auffallend viele Akademiker in Führungspositionen der Wirtschaft und Industrie

1926-1928 Flakhelfer-Jahrgänge ${ }^{75}$

1928 ein überaus erfolgreicher "Starjahrgang"

1929 im Literaturteil der FAZ bezeichnet Frank Schirrmacher die Autoren des Jahrgangs 1929 (Heiner Müller, Günter Kunert, Christa Wolf, Peter Rühmkorff, Hans Magnus Enzensberger, Walter Kempowski, Reinhard Lettau, Michael Ende, Jürgen Habermas u. a.m.) als "die vorläufig letzte homogene Generationsgemeinschaft "(!); in welchem Sinne "homogen“, wäre zu prüfen; vielleicht in dem Sinne, daß sie vor der deutsch-deutschen Teilung wenn auch in West und Ost, doch noch gemeinsam erwachsen wurden

73 Ebd., 116, Herrorhebungen von UH. - Plessner setzt hinzu, daß Mannheim diesen Mechanismus und diese Konsequenz nicht gesehen habe; und dies stimmt überein mit den weiterführenden Überlegungen bei Petersen, Radikale Jugend.

74 Jürgen Eick (Wirtschaftsjournalist, seit 1963 Mitherausgeber der FAZ) beschrieb in der FAZ die Jahrgänge 1920 bis 1930. 1920: 21. 2. 1980, 1921: 10. 2. 1981, 1922: 5. 3. 1982, 1923: 21.2. 1983, 1924:29. 2. 1984, 1925: 6. 2. 1985, 1926: 6. 2. 1986, 1927: 13.2. 1987, 1928: 12.2. 1988, 1929:31. 1. 1989, 1930:6. 1. 1990. Jürgen Jeske (derzeit Mitherausgeber der FAZ) setzte diese Reihe bis zum Jahrgang 1941 mit ausführlichen Namenslisten fort. 1931: 6. 2. 1991, 1932: 7. 1. 1992, 1933: 16.1. 1993, 1934: 11. 1. 1994, 1935: 23. 12. 1994, 1936: 23. 12. 1995, 1937: 27. 12. 1996, 1938: 31. 12. 1997, 1939: 30.12. 1998, 1940: 29. 12. 1999, 1941: 23.12. 2000. - Da es sich um Führungspersonal aus Wirtschaft und Industrie handelt, dienen nur männliche Karrieren zur Charakteristik der Jahrgänge. Erst in den späteren Jahren tauchen auch Frauen aus der Politik auf. Bei den Bemerkungen zu diesen Jahrgängen und den von ihnen gebildeten Kohorten muß dieser Umstand stets beachtet werden, ebenso der Hinweis von Friederike Hassauer (Wien), daß weibliche Generationsbildungen durch die anderen zeitlichen Strukturen weiblicher Lebensentwürfe und -vollzüge (in Ehe, Familie, Beruf, Karriere usw.) zeitlich anders strukturiert sind.

${ }^{75}$ Rolf Schörken, Schülersoldaten - Prägung einer Generation, in: Rolf-Dieter Müller, HansErich Volkmann (Hrsg.), Wehrmacht. Mythos und Realität (München 1999) 456-473; ders., Sozialisation inmitten des Zusammenbruchs. Der Kriegseinsatz von 15- und 16-jährigen Schülern bei der deutschen Luftabwehr (1943-1945), in: Dittmar Dablmann (Hrsg.), Kinder und Jugendliche in Krieg und Revolution (Krieg in der Geschichte, Bd. 7, Paderborn 2000), 123-143. 
1931-1933 Höhere Schule und Ausbildung in den schlechten Nachkriegsjahren und Anfang der 50er Jahre; Augenzeugen des Wirtschaftswunders; in den 80er Jahren Chancen durch Ablösung früherer Jahrgänge

1934

1936

1941

als Jugendliche einer besonderen ${ }_{n}$ Amerikanisierung “ ausgesetzt "Geboren im Jahr der deutschen Bankenkrise" "Nach dem Krieg bekamen viele eine Chance" "In einem Schicksalsjahr geboren: Jahrgang 1933. Mit Sechzig Abschied von der

„Vor neuen Herausforderungen: Jahrgang 1934. Schlankere Unternehmen brauchen weniger Manager"; Aufwachsen im geteilten Deutschland und im Wirtschaftswunder; Rezessionen der 70er und 80er Jahre als berufliche Bedrohungen "Vor einem neuen Lebensabschnirt: Der Jahrgang 1935. Berufswege zwischen Wirtschaftswunder und Strukturwandel"; erfolgreiche berufliche Einstiege in den 70er Jahren; in den 90er Jahren im Osten durch den Umbruch und im Westen durch die Rationalisierungsschübe berufliche Abbrüche

„Fünf Lebensjahrzehnte Frieden: Der Jahrgang 1936. Runde Geburtstage in einer Zeit neuer Herausforderungen “

„Geprägt durch die fünfziger Jahre: Der Jahrgang 1937. Lebenswege, die in einer Zeit beispiellosen Wachstums begonnen haben ${ }^{\text {" }}$

"Und läuft und läuft und läuft: Jahrgang 1938. Nur in Westdeutschland eine Jugend in der Blütezeit des Wirtschaftswunders"

"Zeitzeugen eines zu Ende gehenden Jahrhunderts: Jahrgang 1939. Lebensläufe mit einer Kindheit im Krieg und einem Alter im Frieden"

"Ein dritter Lebensabschnitt im 21. Jahrhundert: Der Jahrgang 1940. Lebensläufe mit mehr als fünfzig Jahren Frieden“

nZeitzeugen tiefgreifender Umwälzungen: der Jahrgang 1941. Lebensläufe zwischen der Zerstörung und der friedlichen Einigung Europas“

(2) Benachbarte Jahrgänge lassen sich zu Kohorten gruppieren. Mehr noch: Innerhalb der benachbarten Jahrgänge lassen sich unterschiedliche Kohorten bilden: Kohorten der Geburtsjahrgänge, der Schulabschlüsse (in den verschiedenen Schulformen), der Berufsausbildungswege, des Eintritts ins Berufsleben, der Erstwähler, in ländlichen oder städtischen Milieus, männlich/weiblich usw. Der Angehörige eines Jahrgangs kann also mebreren Kohorten angehören, jeweils bezogen auf die Fragestellung, zu deren Beantwortung in der empirischen Sozialforschung eine Kohorte gebildet worden ist.

(3) Eine Generation hingegen ist eine Gruppe oder „Gemeinschaft", die sich durch gemeinsame Überzeugungen und „vitale Akzente“ auszeichnet, verbunden vornehmlich nicht durch gemeinsame Lebenserfahrungen (die teilt sie mit unzähligen anderen Zeitgenossen), sondern durch daraus gewonnene Lebensformen und Lebensstile (Habitus, „Prägnanzbildung ${ }^{476}$ ), durch „Lebenshorizonte ${ }^{*}$ und ein bestimmtes „Verhältnis von Gleichzeitigkeit“, das auch altersunabhängig sein kann (Gleichzeitigkeit als solche konstituiert nur Jahrgänge oder Kohorten). Insofern ist die Redeweise z. B. von der Flakhelfer- "Generation “ nicht korrekt, es sei denn, diese Jahrgänge zeigen als Kohorte Verhaltens- und Einstellungseffekte, die sie von anderen signifikant unterscheiden ${ }^{77}$, so daß man sie im Sinne von Diltheys „Prägungshypothese" als Generation bezeichnen kann.

${ }^{76}$ Hinweis von Lutz Niethammer (Jena) im Anschluß an Karl Mannheim.

77 In Schelskys "skeptischer Generation“ finden sich natürlich viele Flakhelfer. Die klassi- 
Mit anderen Worten: Eine "Generation" ist eine Gruppe von Menschen, die sich durch eine für sie spezifische kollektive Identität auszeichnet und sich durch diese miteinander bedeutungsvoll verbunden weiß ${ }^{78}$. Diesen Sachverhalt bezeichnet der Begriff "Generationalität". Eine "Generation" bildet also-in den Worten von Karl Mannheim - aufgrund gemeinsamer Generationslagerung, in jedem Fall aber innerhalb eines Generationszusammenhangs eine Generationseinheit. In der Regel benötigen solche Gruppen, Bewegungen, Generationen „Führer “ (die in der Regel etwas älter sind) und machen sich sozial, politisch, kulturell bemerkbar als kollektive Akteure. "Generation“ meint also nicht im umgangssprachlichen Verständnis Altersabstand, Altersgruppenzugebörigkeit oder Verhältnis von Altersgruppen, sondern eine "Gemeinschaft", verbunden durch spezifische gelebte Werte und Ziele. Dies macht darauf aufmerksam, daß - ähnlich wie bei den Kohorten - die Formierung von "Generationen“ unterschiedliche Anlässe und Konkretisierungen und damit unterschiedliche Funktionen haben kann. Deshalb können „Generationen“ z.B. als "Schicksalsgemeinschaften“ nur wenige Jahrgänge umfassen (die "Flakhelfer"-, die "skeptische Generation“) oder als „Bewegung" Menschen ganz unterschiedlicher Lebensalter miteinander verbinden (die „Grünen“), sie können in Zeiten von geschichtlicher Kontinuität nur gelegentlich hervortreten und für Zeiten von Beschleunigung und zunehmender Dynamik in Verbindung mit besonderen Ereignissen für kurze Intervalle konstruiert werden, wie dies für die Jugend- „Generationen “ vor dem Ersten Weltkrieg geschehen ist ${ }^{79}$.

sche Studie für dic Analyse der Langzcitwirkungen generationsspezifischer Erfahrungen ist Glen H. Elder, Children of the Great Depression. Social Change in Life Experience (Chicago 1974, Boulder $\left.{ }^{25} 1999\right)$.

${ }^{78}$ Kirstin Platt, Mibran Dabag (Hrsg.), Generation und Gedächtnis. Erinnerungen und kollektive Identität (Opladen 1996).

${ }_{79} \mathrm{Vgl}$. Wilhelm Flitner, Die junge Gencration im Volke (zuerst 1928), wiederabgedr. in: ders., Reformpadagogik und Jugendbewegung (Gesammelte Schriften, Bd. 4, hrsg. von Ulrich

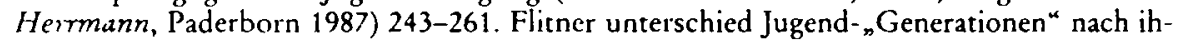
rer „inneren Lage ${ }^{*}$ zu den prägenden Ereignissen ihrer formativen Lebensphase: (1) die um 1890 Geborenen, die Träger der Jugendbewegung, geprägt vom Geiste Nietzsches und der Neu-Romantik; (2) die um 1895 Geborenen, deren "geistiges Erwachen“ in die Kriegsjahre fiel, mit entsprechenden Orientierungs- und Einmündungsproblemen unmittelbar nach dem Krieg; (3) die zwischen 1896 und 1904 Geborenen, in deren Jugendzeit noch der Krieg und die demoralisierende Nachkriegszeit (bis um 1925) fiel; (4) die Gruppe der zwischen 1905 und 1912 Geborenen, deren Jugend und formative Lebensphase zusammenfiel mit dem Untergang der Republik und dem Aufstieg des Nationalsozialismus. Flitner selber war 1889 geboren worden und lag seiner Herkunft nach vor diesen Generationen (dazu ders., Autobiographie: Erinnerungen 1889-1945 [Gesammelte Schriften, Bd. 11, Paderborn 1986); er betrachtete die Abfolge seiner "Generationen" aus dem Blickwinkel ihrer "Lagerung“ in der Nachkriegszeit. Eine andere geläufige Trennlinie ist auch der Jahrgang 1902, der letzte, der noch regulär zum Kriegsdienst herangezogen wurde. Vgl. Emst Glaeser, Jahrgang 1902 (Potsdam 1928). - Eine ähnliche Abgrenzung von Generationen geht auf Eduard Spranger zurück: „die“ Vorkriegsgeneration, die um 1900 im Alter von 20 halbwegs erwachsen war; die Generation der Jugendbewegung; die bündische Jugend; Jugend im Nationalsozialismus; Jugend nach 1945, in: Eduard Spranger, Fünf Jugendgenerationen 1900-1949, in: ders., 


\section{2) Typologie und Funktionen des „Generations"-Konzepts}

Dilthey sah die "Generation" der Romantiker in der Abhebung von den gleichzeitig lebenden und wirkenden Dichtern und Philosophen der Klassik. Petersen analysiert ein bestimmtes politisch radikalisiertes Segment der sozialistischen Jugendbewegung in der Schweiz. Fogt rekonstruiert die Generationseinheit der " $68 \mathrm{er}^{\text {" }}$. Hier sei - nicht zuletzt aufgrund der Anregungen bei der hier dokumentierten Konferenz - der Versuch gemacht, Typen und Funktionen in einer gewissen Systematik vorzustellen.

Typen von "Generationen " ergeben sich

(a) aus der Perspektive ibrer Prägung oder ibrer Konstruktion: geprägt durch das Kriegserlebnis, die Nähe des Todes, Eltern- und Heimatlosigkeit, allgemeiner: durch existentielle unauslöschliche Erfahrungen ${ }^{80}$; als Selbstbeschreibung ( ${ }_{n}$ Das junge Deutschland“), als Selbst- und Fremdzuschreibung ("die 68er ${ }^{\text {) }}$, als retrospektive soziologische Rekonstruktion (die ${ }_{n}$ skeptische Generation“). Dabei kann

(b) durch die Konstruktion von Idealtypen ("Protestgeneration") oder durch phänomenologische Deskription ("Beat-Generation“) oder durch "dichte Beschreibung" von "Gestalten " („der Wandervogel“) vorgegangen werden. Des weiteren können

(c) Lebensentwürfe und -erwartungen sowie die Abfolge der Altersgenerationen im Kontext Sozial-kulturellen Wandels unterschiedliche Generationenbeziebungen formulieren: Kontinuität und Diskontinuität, Innovation (Dynamik) und Traditionalität (Beharrung), Spannungen und Konflikte ${ }^{81}$; das Problem der Generationsverträge und der gegenseitigen Solidarleistungen ${ }^{82}$.

Daraus ergeben sich unterschiedliche Funktionen unterschiedlicher "Generations"-Konzepte. "Generationen" können Gruppen und Gemeinschaften sein für spezifische

Pädagogische Perspektiven (Heidelberg 1951) 25-56; Spranger beschreibt offensichtlich Generationslagerungen. - Jürgen Zinnecker (Siegen) wies auf die Differenzierung von Alters-, Kohorten- und Ereigniseffekten hin.

${ }^{80}$ Bernd Giesen (Konstanz) hat darauf hingewiesen (vgl. seinen Beitrag in diesem Band), daß es sich dabei dann um Erfahrungen handelt, die in der Regel nicht mit den Deutungsmustern der älteren oder einer anderen Generation bewältigt werden können.

81 Wobei zu beachten ist, daß "Generationsgenossen" sich auch auf ein gemeinsames in sich Gegensätzliches beziehen können (Lutz Niethammer mit Hinweis auf Mannheim): die Jugendbewegung vor dem Ersten Weltkrieg war z. B. eine „rückwärtsgewandte Modernisierung".

82 Diesem Sachverhalt gelten die Arbeiten des Konstanzer Soziologen Kurt Lüscher (und seiner Arbeitsgruppe): Generationenbeziehungen sind historisch zunächst und vor allem juristische Beziehungen (Erbrecht), in denen zugleich soziale und ökonomische Machtpositionen definiert und weitergegeben oder vorenthalten werden. Kurt Lüscher, Franz Schultheis (Hrsg.), Generationenbeziehungen in "postmodernen" Gesellschaften (Konstanzer Beiträge zur sozialwiss. Forschung, Bd. 7, Konstanz 1993). - Vgl. dazu den Beitrag von Gerd Hardach (Marburg) in diesem Band. 
(a) Kommunikations- und Verständigungsprozesse: die Gruppierungen der Jugendbewegung und der bündischen Zeit, Künstlerkolonien und ihre Sezessionen, Wissenschaftler-Gruppen bei Paradigmen-Wechseln, nachrückende Politikergruppen (die Generation der „Enkel“ Konrad Adenauers), die „Golf-Generation“ der Yuppies, unterschiedliche "Medien-Generationen";

(b) Deutungs- und Erinnerungsarbeit: Vereinigungen zur Traditionspflege, darin der insofern gar nicht kuriose Fall der nachträglichen Selbstzuschreibung zu den „68ern“; Versuche, „Hegemonial-Tendenzen“ und damit sich selber als Gruppe bzw. Gemeinschaft durchzusetzen ${ }^{83}$;

(c) Aktions- und Wirkungsformen: die Weiße Rose, „die Studentenbewegung “ 1967 ff.; diese Gruppen und Gemeinschaften bilden "Problemgemeinschaften“ oder auch „Problemlösungsgemeinschaften“ (in der SBZ/DDR z.B. die FDJ"Aufbaugeneration“).

Für diese unterschiedlichen „Generationen“ gibt es unterschiedliche „Bindekräfte" und "Bindemittel " (Plessner), und diese sind es, mit deren Hilfe wir "Ge-

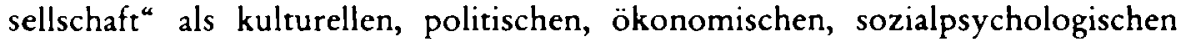
„Wirkungszusammenhang “ von Gruppen und sozialen Formationen entziffern und „lesen". Wenn wir daher mit den entsprechenden inhaltlich "gefüllten" Generationenbegriffen die "Wirkungszusammenhänge“ der geistigen und sozialen Welt entziffern (Dilthey), erzeugen wir geistes-, kultur- und sozialwissenschaftliches Deutungswissen und sozial-kulturelle Ordnungsmuster für Vergangenheiten und Gegenwarten. Geschichte in "Generationen" zu denken ist zwar kein geeignetes Mittel der Periodisierung ${ }^{84}$, wohl aber ein überaus ergiebiger Ansatz einer „erweiterten Sozialgeschichte ${ }^{* 85}$.

\section{3) Die "Generation der Unbefangenen " - keine „Generation“}

Ein Blick auf die Jahrgangscharakteristika hat Gruppierungen von Kohorten gezeigt, und von dort haben wir weiter zu fragen, ob es sich dabei um Übergänge zu Gencrationszusammenhängen oder gar Generationeneinheiten (den „Generationen“ i.e.S.) handeln könnte. Auffallend ist, daß über manche Jahrgänge i.S. ihrer Generationslagerung nichts besonderes zu sagen ist, sondern nur dies: Angehörige bestimmter Jahrgänge hatten durch die Gunst (oder Ungunst) der Umstände besondere Chancen oder Versagungen. Der „leergeschossene" Jahrgang 1922 hatte optimale universitäre Chancen, spätestens seit der Vervielfachung der Lehr-

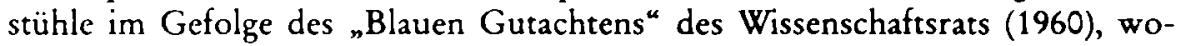
durch seit der Mitte der 80er Jahre ein „Generationswechsel“ in vielen akademischen Disziplinen eintreten $m u ß t e$, mit den entsprechenden Chancen für den

${ }^{83}$ So die Formulierung von Ulrich Herbert (Freiburg). Dies ist die uns inzwischen wohlvertraute Strategie, "Themen zu besetzen " und den "Diskurs" zu instrumentieren und zu instrumentalisieren. Diesem Thema widmet sich der SFB ${ }_{n}$ Erinnerungskultur ${ }^{c}$ der Universität Gießen.

${ }^{84}$ Jäger, Generationen, 451.

85 Ebd., 450. 
Nachwuchs der übernächsten Generation. Oder: Wer 1924/25 geboren worden und 20 Jahre später durch Ausbildung und Studium in gute Startpositionen gekommen war, fand sich 40 Jahre später in Spitzenpositionen. Der "Starjahrgang" 1928 (FAZ) in der Wirtschaft wuchs seit 1948 nach der Währungsreform in das Wirtschaftswunder hinein.

Die Lagerung der Kohorte um 1939 (die ${ }_{n} 38 / 42 e^{\text {c86 }}$ ) läßt sich in folgender Weise skizzieren, wenn (wie oben betont) ein Ausschnitt aus den Geburtsjahrgängen betrachtet wird, der als männlich/westdeutsch/mittelständischer bzw. bildungs-/wirtschaftsbürgerlicher Herkunft, vorwiegend städtisch, weiterfübrende Schulbildung beschrieben werden kann. Die Bildung einer Kohorte mit diesen Milieumerkmalen als Generationszusammenhang ergibt sich aus folgenden Überlegungen: Wer 1937 geboren wurde, gehörte bei der Einführung der Wehrpflicht zu den sog. "weißen Jahrgängen “ und war Mitte/Ende der 50er Jahre mitten im „Wirtschaftswunder" -Aufschwung. Wer 1943 geboren wurde, war bei der Währungsreform erst 5 Jahre alt und kannte in seiner späteren Kindheit und Jugend nur das Wirtschaftswunder und hat auch keine Jugendarbeitslosigkeit mehr erlebt. An den Universitäten konnte er noch mitten im Studium von der Ereignissen 1966 ff. betroffen werden. Wenn hier die Kohorte 1938/42 als Generationszusammenhang vorgeschlagen wird, bedeutet das natürlich nicht, daß sich auch Angehörige weiterer benachbarter Jahrgänge darin wiedererkennen können.

Jürgen Reulecke hat sich mit diesen Jahrgängen der „unbefangenen Genera-

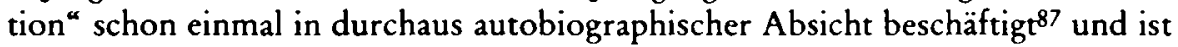
dabei zu einem Ergebnis gekommen, das er nochmals überprüft sehen wollte: „Die um 1940 Geborenen stehen irgendwie, ungenau“ zwischen der profilierteren ,skeptischen Generation' und der noch profilierteren ,68er-Generation'; sie vereinigen vermutlich von beiden Generationsprofilen etwas in sich, und deshalb mögen sie vielleicht so etwas wie eine Brückengeneration sein, deren Lebensthema weder das unkritische Erhalten und Bewahren noch das radikale Ändernund Verbessernwollen ist, sondern das nachdrückliche Reformieren mit Augenmaß. " ${ }^{88}$ Ist diese Selbstzuschreibung zutreffend?

86 Eine Kohortenbildung 1939-1948 (Blücher) erscheint mir problematisch. Jene von Bude (1938-1948) (wie Anm. 40) - und diese auch noch in toto als "68er" zu bezeichnen - ist auch dann nicht begründet, wenn die Interviews einiger Protagonisten kombiniert werden; und die Kohorten in schematischen Dekaden ab 1945 (Jaide, Generationen) liegen quer zu allen historisch wichtigen Kontexten und Ereignissen. Sowohl der mainstream der Kohorten innerhalb der Generationslagerung und des Generationszusammenhangs als auch die Generationseinheiten von $68 \mathrm{ern}{ }^{\star}$ lassen sich (mit der Zustimmung von Wolfgang Kraushaar) mit den entsprechenden Randstreifen zu jeweils 5 Jahrgängen gruppieren (1937/8-1941/2, 1939/ 40-1944/5, 1944/5-1950; letztere vor allem Mitläufer). Kraushaar bezeichnet die „68er “ dezidiert nicht als Generation, was m.E. aber im Lichte der oben skizzierten Funktionstypen von "Generationen" durchaus möglich ist.

87 Jürgen Reulecke, Waren wir so? Zwanzigiährige um 1960: Ein Beitrag zur „Ich-Archäologie ${ }^{*}$, in: Petra Götte, Wolfgang Gippert (Hrsg.), Historische Pädagogik am Beginn des 21. Jahrhunderts. Bilanzen und Perspektiven (Essen 2000) 169-180.

88 Ebd., 180. 
Zunächst ist ein Blick auf Ereignisse und auf kollektiv-biographisch milieubzw. zeitgeschichtsrelevante Daten angezeigt ${ }^{89}$.

Eine Chronik für die Geburtsjabrgänge 1938, 1939, 1940 für das Segment „westdeutsch, männlich, Bildungsbürgertum/Mittelstand, weiterführende Schulbildung ${ }^{\alpha 0}$

\section{- bis 1945 Kriegszeit}

frühe Kindheit, die vollständig in die Kriegszeit fällt, in der Regel vaterlos aufgewachsen, starke Mutterbindung, Halb- und Vollwaisen; Erfahrungen des Krieges gegen die Zivilbevölkerung, Erlebnis des Kriegsendes; Ende einer Familien-Kindheit unter Ausnahmebedingungen; der Jahrgang 1938 wird zum Teil noch eingeschult (Ende 1944 bis 1945/46 unterbrochen); für die nächsten Jahre unterschiedliche Ausgangsbedingungen:

- ausgebombt ja/nein (Wohnung, städtische Infrastruktur, im Westen 2,5 Mio. Wohnungen zerstört)

- umgezogen $j a / n e i n$

- vertrieben $\mathrm{ja} /$ nein $^{91}$

- Groß-/Mittel-/Kleinstadt, Dorf ${ }^{92}$

- soziale Schichtzugehörigkeit

- männlich/weiblich

- 1945/46-1950 unmittelbare Nachkriegszeit, Wiederaufbau und Westintegration

Einschulung mit überfüllten Klassen, Absolvierung der Grundschul-Kindheit, zumeist mangelhafte Grundversorgung; anspruchslose, aber relativ stabile Lebens- und Schulerfahrungen, große Spielräume und unkontrollierte „Spielräume“ auf Straßen und (Trümmer-)Grundstücken ${ }^{93}$

Deutschland unter 4-Mächte-Verwaltung, Errichtung der Länder, Marshall-Plan, Einbeziehung Deutschlands in den Wiederaufbau Europas (von der SU für die SBZ abgelehnt), Währungsreform, Grundgesetz und „Soziale Marktwirtschaft“,

${ }^{89}$ So auch Jaide, Generationen. 25-35. 245-249. Jaide beschreibt und vergleicht für die Zeiträume 1871-1914/18, 1919-1933, 1933-1945 und dann in Dekaden 1945-1955 keine "Generationen", sondern (wie er selber sagt) die "Lagerungen" von Kohorten (299).

90 Man kann auch innerhalb dieser Generationslagerung andere Segmente bilden. Es müssen immer bestimmte Generationszusammenhänge gebildet werden, ohne die es keine Generationseinheiten und keine ${ }_{\infty}$-lücken ${ }^{\alpha}$ geben kann.

${ }_{91}$ Flüchtling zu sein, machte in der unmittelbaren Nachkriegszeit eine enorme Differenz. Vgl. Volker Ackermann, Deutsche Flüchtlingskinder nach 1945, in: Dittmar Dablmann (Hrsg.), Kinder und Jugendliche in Krieg und Revolution (Krieg in der Geschichte, Bd. 7, Paderborn 2000) 145-167; Klaus Naumann (Hrsg.), Nachkrieg in Deutschland (Hamburg 2001).

92 Das ganz andere Profil der zeitgenössischen Landjugend zeigt Ulrich Planck, Landjugend im sozialen Wandel. Ergebnisse einer Trenduntersuchung über die Lebenslage der westdeutschen Landjugend (München 1970).

${ }_{93}$ Roland Gröschel, Michael Schmidt, Trümmerkids und Gruppenstunde. Zwischen Romantik und Politik: Jugend und Jugendverbandsarbeit in Berlin im ersten Nachkriegsjahrzehnt, hrsg. vom Landesjugendring Berlin (Berlin 1990). 
Adenauer 1. Bundeskanzler, Heuss 1. Bundespräsident, 1950 Ende der Lebensmittelrationierung

1949 von ca. 5,5 Mio. deutschen Schulkindern sind 52\% nicht bei beiden Elternteilen (täglicher Suchdienst im Radio, 1950 noch 1,5 Mio. Vermißte)

1950 in Westdeutschland leben ca. 1 Mio. Witwen und 1,3 Mio. Waisen, davon 1,25 Mio. vaterlos, 30000 Vollwaisen

Ende der Grundschule, Übergang auf weiterführende Schulen (nur ca. 5\% dieser Jahrgänge kommen zum Abitur)

-1950-1955/56 frübe Jugend: Beginn der Ära Adenauer, „Wirtschaftswunder ${ }^{\alpha}$

"Wirtschaftswunder “ (Index BRD $1950=100$ )

$\begin{array}{lll} & 1951 & 1955 \\ \text { Industrie-Produktion insgesamt } & 118 & 178 \\ \text { Grundstoff-Produktion } & 118 & 174 \\ \text { Investitionsgüter-Produktion } & 131 & 233 \\ \text { Verbrauchsgüter-Produktion } & 114 & 162 \\ \text { Lebenshaltungskosten } & 108 & 110 \\ \text { durchschnittl. Bruttoverdienst/Woche } & 113 \mathrm{DM} & 144 \mathrm{DM}\end{array}$

Einkommen Privathaushalte BRD (in Mrd. DM)

$\begin{array}{llllll}1951 & 67,8 & 1954 & 88,6 & 1956 & 108,4\end{array}$

1950 Krieg in Indochina und Korea, China besetzt Tibet (bis heute)

1951 westliche Alliierte beenden Kriegszustand mit der BRD, Verhandlungen über westdeutsche Wiederbewaffnung, Suez-Krise

1952 Montan-Union, Lastenausgleichs-Gesetz, de-facto-Beendigung der Verfolgung von NS-Verbrechern, Verschärfung der deutschen Teilung, Fluchtwellen aus der DDR, deutsch-israelisches Wiedergutmachungsabkommen

1953 Londoner Schuldenabkommen, 2. Kabinett Adenauer, 17. Juni (Fluchtwellen), Stalins Tod

1954 Berliner Deutschland-Konferenz der Siegermächte ohne Ergebnis, Beendigung des Besatzungsregimes, Pariser Verträge (Saar-Regelung), Ende des Indochina-Kriegs, Beginn des Algerien-Kriegs

1955 Souveränität der BRD, Adenauer in Moskau, atomare Aufrüstung (HBombe)

1956 Wehrpflicht, Gründung der Bundeswehr, XX. Parteitag der KPdSU, Ungarn-Aufstand, KPD-Verbot

neues Spielzeug, Lieblingsautoren von Mädchen: Spyri, Defoe, Ury, Kästner; von Jungen: Karl May, Defoe, Löns, Twain, Kästner; Schulfunk, erste Fernsehprogramme (1952 des NWDR), neue Konsumgüter, erste Ferienreisen

die individuelle Entwicklung vollzieht sich in Übereinstimmung mit den wahrnehmbaren familialen und sozialen Lebensformen ( angepaßtes Verhalten ${ }^{\text {) }}$; zugleich eingestellt auf und gefördert hin zu Selbständigkeit; die eigenen Jahrgangsgemeinschaften der Gymnasiasten leben ohne eigene markante Codes oder erkennbare (Selbst-)Identifikationssysteme 
die nächst ältere Halbgeneration (Geschwister) ist im Arbeitsleben verschwunden; die Generation der Eltern und Lehrer bietet kaum Anlässe für (politische) Konfrontation oder Distanzierung; wenig wahrnehmbare Indoktrination, gepaart mit Politikferne; eine sich konsolidierende Demokratie mit heftigen Parlamentsdebatten ist die einzige und damit ${ }_{n}$ normale ${ }^{*}$ Politikerfahrung

1955 (bis 1958) „Halbstarke“: Jugendkrawalle der ab 1940 geborenen Arbeiterjugendlichen ${ }^{94}$ (Zinnecker: ein anderer Generationszusammenhang im Vergleich zu den Protagonisten der '68er'5)

-1955/56-1960/61 Ende der (verlängerten) Jugend-und Schulzeit (Gymnasium, Abitur)

Schul- und Jugendzeit fällt komplett in die Zeit des "Wirtschaftswunders" und die konsolidierte Ära Adenauer

Stabilisierung der äußeren Verhältnisse durch die politische und wirtschaftliche Konsolidierung Westeuropas sowie durch die Berliner Mauer nach Osten (Kalter Krieg als Stabilisierung), "Kampf dem Atomtod“ und Ostermarsch-Bewegungen (der Jahrgänge um 1928)

1957 3. Kabinett Adenauer, „Göttinger Appell“ gegen die Atomrüstung der Bundeswehr

1958 Beginn der Berlin-Krise, De Gaulle beendet die 4. Republik, deutsch-französische Aussöhnung

1959 Godesberger Programm der SPD, Willy Brandt Bürgermeister von Berlin, Gemeinsamer Markt, Vollbeschäftigung (weniger Arbeitslose als offene Stellen), Koexistenz-Politik der Großmächte, Fidel Castro siegt auf Cuba

1960 Algerien-Krise, Ulbricht Staatsratsvorsitzender, 200000 Flüchtlinge aus der DDR (davon $49 \%$ unter 25 Jahre)

1961 im Juni 30000 DDR-Flüchtlinge, bis September 196000; am 13. 8. Bau der Berliner Mauer, 4. Kabinett Adenauer

Auslandsferienreisen, Führerschein; „Twen“ erscheint 1958 (die Abiturienten fühlten sich nicht als Twens, und die gleichaltrigen Mädchen trugen auch keine Petticoats mehr); feste Partnerschaften (in der Regel durch das gemeinsame Gymnasium oder in engen lokalen Freundes- und Vereinszirkeln); kaum politische Bindungen oder Engagements, kirchliche bzw. religiöse Bindungen; selbstbewußte pragmatisch-optimistische Grundeinstellungen ohne nennenswerte Konflikterfahrungen

Beendigung der weiterführenden Schulen, offene Studien- und Ausbildungsmöglichkeiten (Förderung nach dem „Honnefer Modell“ seit 1957), offene Arbeits-

94 Günther Kaiser, Randalierende Jugend. Eine soziologische und kriminologische Studie über die sogenannten ${ }_{n}$ Halbstarken" (Heidelberg 1959).

${ }^{95}$ So sein Beitrag: "Halbstarke“ - die andere Seite der 68er-Generation, in: Ulrich Herrmann (Hrsg.), Protestierende Jugend. Jugendopposition und politischer Protest in der deutschen Nachkriegsgeschichte (Weinheim, München 2002) 461-485. 
märkte, offene Lebenschancen; Wehrdienst, Studienbeginn (völlig überfüllte Universitäten in Altbauten mit wenigen Professoren)

-1960/61-1968 Berufsausbildung und Studium, Eintritt ins Berufsleben, Ende der Ära Adenauer, Ende des „Wirtschaftswunders"

1962 „Spiegel“-Affäre, 5. Kabinett Adenauer

1963 Rücktritt Adenauers, Erhard wird Bundeskanzler, Auschwitz-Prozeß in Frankfurt a.M. (bis 1965), Kennedy wird ermordet

1964 1. Wirtschaftskrise, Vietnam-Krieg, Anfänge der APO ${ }^{96}$

1965 Georg Picht: „Die deutsche Bildungskatastrophe“; Beginn der Bildungsexpansion und der Gesamtschulversuche, Demonstrationen gegen den Bildungsnotstand, Universität Bochum wird eröffnet; 2. Kabinett Erhard

1966 Große Koalition (Kiesinger/Brandt), Vietnamkongreß in Berlin, RassenUnruhen in den USA, "Kulturrevolution " in China, (bildungs-)politische Konflikte an den Universitäten, Beginn der Untersuchungen zur „braunen Universität"; Eröffnung mehrerer Universitätsneugründungen

Von Friedeburg u. a. ${ }^{97}$ ermittelten das politische Potential der Studierenden der FU Berlin:

demokratisch $26 \%$ (davon definitiv $13 \%$, tendenziell $13 \%$ )

unprofiliert $62 \%$ (davoneber demokratisch $13 \%$, ganz unprofiliert $19 \%$, disparat $10 \%$, eher autoritär $20 \%$ )

autoritär $\quad 12 \%$ (davon tendenziell $6 \%$, definitiv $6 \%$ )

$1967 \mathrm{ff}$. Beginn Studenten- und Schülerbewegung, Nullwachstum des Bruttosozialprodukts: Ende des „Wirtschaftswunders“

1967 Tod Adenauers, Benno Ohnesorg wird bei den Anti-Schah-Demonstrationen in Berlin erschossen

1968 Notstandsverfassung, Attentat auf Rudi Dutschke, weltweit Studentenunruhen, Beginn der inneren Hochschulreform; Intervention der UdSSR in der CSSR

Ende des Studiums, Eintritt ins Berufsleben; kaum Politisierung im Vorfeld von 1968, wenig direktes (partei-)politisches Engagement nach 1968; Orientierung an den individuellen Lebensentwürfen, frühe Eheschließungen; beruflicher Aufstieg im Kontext der gesamt-gesellschaftlichen Liberalisierungstendenzen, Funktionen in mittleren und Führungspositionen: pflichtbewußt, pragmatisch, kreativ, wenig hierarchiebetont; Leben in Familien- und Freundeskreisen, Kinder- und Jugend-

$96 \mathrm{Zu}$ den Protesten im Vorfeld der Ereignisse von 1967/68 vgl. die Dokumentationen von Kraushaar (wie Anm. 38) und Hermann, Protestierende Jugend, dort die Beiträge der Abteilung „Gegen die Remilitarisierung und „Kampf dem Atomtod!“

${ }^{7}$ Ludwig von Friedeburg u.a., Freie Universität und politisches Potential der Studenten. Über die Entwicklung des Berliner Modells und den Anfang der Studentenbewegung in Deutschland (Soziologische Texte 57, Neuwied, Berlin 1968). 
leben der eigenen Kinder, Sicherung des Lebensstandards auf Wohlstands-Niveau ${ }^{98}$.

Mit dem Eintritt in ihr viertes Lebensjahrzehnt können wir die zeitgeschichtlichen Umstände der „38/42er “ verlassen. 1969 hatte der Arbeitsmarkt 860000 offene Stellen bei 1,5 Mio. Gastarbeitern. Die private berufliche Etablierung erfolgte ohne Schwierigkeiten, trotz nachfolgender Wirtschaftskrise. Politik war und blieb Nebensache, in präziser Differenz zu denen, bei denen die Ereignisse um 1968, die Bewegung der Grünen, die Friedensbewegung und die Nachrüstungsdebatte eine Politisierung bewirkt hatten.

\section{4) Die "Unbefangenen " und die „unauffällig Integrierten" in der Generationen- ${ }_{n}$ Lücke ${ }^{\alpha}$}

Hinsichtlich ihrer Generationslagerung befanden sich die „Unbefangenen“ (Blücher) bzw. „unauffällig Integrierten“ (Pfeil/Friedrichs) zwischen der „Flakhelter-“ und der "skeptischen Generation“ auf der einen und der Generation der "68er" auf der anderen Seite. Die 1938/39 und später Geborenen waren bei Kriegsende Kinder. Von prägenden Ereignissen und Erlebnissen im Krieg und bei Kriegsende ist bei dem Ausschnitt aus dieser Kohorte, der hier betrachtet wird, vielfach zu berichten; aber sie blieben häufig unterschwellig und nicht immer manifest wirksam, weil bei dem größeren Teil dieser Jahrgänge eine Erfolgsgeschichte sie überlagerte: Die späte Kindheir fiel zwar in die unmittelbare Nachkriegszeit, diese zeichnete sich aber zunächst und vor allem durch die Normalität der jetzt beginnenden Schulzeit aus. Die Jugendzeit und zwei Drittel der Schulzeit fielen in die Zeit des sich konsolidierenden politischen Systems der Bundesrepublik und

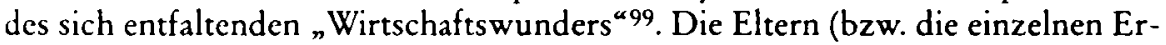
ziehungsverantwortlichen) waren durchweg mit Wiederaufbau beschäftigt. Die Lehrerschaft war in politischer Hinsicht durch innere Verwerfungen paralysiert: neben den wenigen aufrechten NS-Regime-Fernen (der "gebildete " $P$ hilologe*, der mit dem „braunen Pöbel“ nichts im Sinn gehabt hatte und eher unpolitisch war) gab es „Mitläufer" und „Belastete “ (letztere waren zum 'Teil erst nach der Revision der Beamtengesetze Anfang der 50er Jahre wieder in den Schuldienst zurïckgekommen), und es gab junge Lehrer, die Krieg und zum Teil Gefangenschaft überlebt hatten und in die Normalität einer bürgerlichen Existenz strebten. Alle diese Gruppen hatten mit den „38/42ern “ politisch nichts im Sinn: die erste Gruppe war nicht politisch; die zweite hütete sich davor, es zu sein; und die dritte

98 Josef Ehmer (Salzburg) mußte über soviel Selbstbekenntnis schmunzeln und fühlte sich lebhaft an gleichaltrige Universitätskollegen erinnert. Heinz Bude (Berlin) war regelrecht erschrocken über eine so "langweilige Generation“. Für Lutz Niethammer war dies Anlaß für den Hinweis, es wäre doch sicherlich aufschlußreich, einmal den lebensgeschichtlichen Daten und Erfahrungsbereichen des "Langweiligen" und "Spannenden" nachzugehen und so über eine biographie intime noch ganz andere Dimensionen und Charakteristika von Generationszugehörigkeiten zu entdecken.

99 Vgl. Preuß-Lausitz u.a., Kriegskinder (wie Anm. 46). 
"hatte die Schnauze voll ${ }^{\alpha}$. Mit einigen wenigen Geschichtslehrern fand die Beschäftigung mit der NS-Zeit statt, denn es gab ja bereits die Dokumentationen von Hofer ${ }^{100}$. Die "Halbstarken"-Krawalle in der Mitte der 50er Jahre gingen an den Gymnasiasten vorbei, für die Anti-Atom- und die Ostermarsch-Bewegungen waren sie zu jung und zu unpolitisch, für ${ }_{n} 68^{\star}$ zu alt. Es gab keinen Numerus clausus, mithin konnte der Tauschwert der Abiturnoten unberücksichtigt bleiben und führte zu durchweg mäßigen Leistungsstandards; dafür gab es hoffnungslos überfüllte Hörsäle und Seminare. Der Arbeitsmarkt erlaubte Ferienarbeit für die ausreichende Selbstfinanzierung des Studiums, nach den Examina standen praktisch alle beruflichen Möglichkeiten und Karrieren offen. Die politische Sozialisation erfolgte unter den Bedingungen eines Systems, das offensichtlich gut funktionierte, das kontroverse Standpunkte zuließ, keine Loyalitätskonflikte heraufbeschwor, als "Parteiendemokratie" kein bürgerschaftliches Engagement herausforderte, sich durch eine beruhigend wirkende Großväter-Generation repräsentierte (Adenauer, Heuss) und als Wirtschaftssystem in eine unaufhörlich wachsende Wohlstandsgesellschaft mit Vollbeschäftigung führte, während sich der „real existierende Sozialismus" durch Massenflucht und Mauerbau delegitimiert sah; mit dem Godesberger Programm und den Bildungsreformdebatten wurde bereits in den frühen 60er Jahren Reformwilligkeit und -fähigkeit signalisiert.

Als an den Universitäten Unruhe ausbrach, hatten die Jahrgänge 1938/42 sie durchweg bereits verlassen oder schrieben an ihren Doktorarbeiten. Die "38/ $42 \mathrm{er}^{\text {" }}$ waren keine aktiven "68er ${ }^{\text {, }}$, bis auf einige wenige "Führer" in diesen Jahrgängen, ohne die es allerdings keine „Bewegung“ und "Gefolgschaft" der Jüngeren gegeben hätte: Rabehl wurde 1938 geboren, Kunzelmann 1939, Dutschke 1940, Langhans 1941.

\section{Zwischen „Halbstarken" und „68ern": der unaufhaltsame Aufstieg der „Unbefangen-Unauffälligen“}

Blüchers "Generation der Unbefangenen“ und Pfeils „23jährige“ beschreiben keine "Generationen", die im Jugendalter in dem Sinne eine spezifische Prägung erhalten hätten, daß dies zu "Prägnanzbildung “ und zu „vitalen Akzenten“ (Plessner) von Gruppen als Generationseinheiten geführt hätte. Vielmehr handelt es sich bei diesen benachbarten Jahrgängen um eine Kohorte, die auch „Zwischengeneration ${ }^{\prime 101}$ genannt wurde. Geprägt wurde sie nicht durch herausragende Er-

100 Walther Hofer (Hrsg.), Der Nationalsozialismus. Dokumente 1933-1945 (Frankfurt a.M. 1957, noch im Erscheinungsjahr im 100.-150. Tsd., 1994 im 1071.-1076. Tsd.!!); ders. (Hrsg.), Die Entfesselung des Zweiten Weltkrieges (Frankfurt, Hamburg 1960, u.ö.).

101 Jaide, Generationen, 285: „Zwischengenerationen ${ }^{\text {, }}$, die gleichsam in der $W_{1}$ indstille der Geschichte mit stillen Spärlingen oder Vorläufern oder als Statthalter für die Folgenden wir$\mathrm{ken}^{\circ}$. Richtig ist, daß die „38/42er “ "gleichsam zwischen profilierteren Hauptgenerationen“ aufwuchsen. Es handelt sich auch nicht um eine "verschlissene* Zwischen- oder um eine ${ }_{n}$ Epigonen“-Generation (ebd.). 
eignisse und Erfahrungen in ihrer formativen Phase $z$ wischen 14 und 24, sondern geformt durch die allgemeinen Lebensverhältnisse, die - wie oben angedeutet - in mehrfacher Hinsicht begünstigend gewesen sind. Alle konnten bemerkenswerte berufliche Chancen wahrnehmen und schließlich auch alle sozial-kulturellen und politischen Emanzipationszuwächse im Gefolge der „68er-Kulturrevolution“ beerben: die „38/42er" sind in mehrfacher Hinsicht Gewinner gewesen! Ihre Formung geschah als Anpassung, Funktionieren, Vorwärtsstreben, wie es bei Pfeil/ Friedrichs beschrieben ist, und diese Formung geschah wirksam durch die Erfahrung ihrer positiven Effekte im Lebensalltag. Deshalb: Worüber hätten sich diese Jahrgänger beklagen, wovon sich distanzieren sollen, wogegen sich politisch zur Wehr setzen müssen? Es gab durchaus Ereignisse, mit denen man sich auseinandersetzen mußte: der 17. Juni 1953, der Ungarn-Aufstand und seine Niederschlagung 1956, die Wiederbewaffnung, der Mauerbau 1961. Aber schlußendlich hatte der "freie Westen" und seine Eindämmungspolitik, d.h. hatte die Zementierung der deutschen Teilung und des Kalten Krieges für ungestörte Sicherheit und zunehmenden Wohlstand gesorgt.

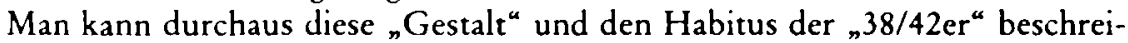
ben, aber sie sind keine "Generation", es sei denn, man wollte sie die erste "Wirtschaftswunder-Generation" nennen. Für diese Kohorte ist der Ausdruck "Zwischengeneration" (Jaide) unglücklich, weil dadurch die begriffliche Klarheit des oben entwickelten Generationsbegriffs wieder verschwimmt. Und da "Nicht" Generation ein leerer Un-Begriff ist, liegt es nahe, wieder auf die Mannheimsche Bezeichnung Generationszusammenhang zurückzukommen. Dieser Generationszusammenhang ist charakterisiert durch die skizzierte Generationslagerung am Kriegsende und in der Nachkriegszeit, dann aber vor allem für die Jugendzeit als der formativen Phase im sich entfaltenden "Wirtschaftswunder". Deshalb könnte mit einem gewissen Recht von der ersten "Wirtschaftswunder-Generation" gesprochen werden, in der "Lücke" zwischen der "skeptischen Generation" und den "68ern" und in dieser "Lücke" neben der "Generation" der "Halbstarken“. Insofern kann man - mit Jürgen Reulecke - nicht von einer „Brückengeneration" sprechen, denn was wäre ihre Brückenfunktion gewesen? Da wäre schon "Übergangsgeneration ${ }^{*}$ richtiger. Aber noch einmal: die hier beschriebenen "38/42er" sind keine ,Generation"!

Gleichwohl ist im ganzen wohl Jürgen Reuleckes oben zitierte Charakteristik der um 1939 Geborenen in dem hier betrachteten sozial-kulturellen und beruflichen Segment zutreffend, weil die ${ }_{n} 38 / 42 \mathrm{er}^{*}$ habituelle Züge aus den Zeiten vor und nach der Epochenschwelle von, $68^{\circ}$ zeigen: in Konventionen und Institutionen denkend und zugleich experimentierfreudig, in der Wissenschaft an herkömmlichen Standards orientiert und zugleich neue Fragestellungen generierend und auslotend, im persönlichen Leben eher traditionelle Lebens- und Familienformen pflegend obne Schwierigkeiten mit den anderen Lebensstilen der Kinder. Vermutlich ein weiterer Grund dafür, daß sich nachträglich viele Angehörige dieser Kohorte den ${ } 68 \mathrm{ern}{ }^{\star}$ zuschreiben und mit einem gewissen Recht auch als einer .Erinnerungsgeneration“ zuschreiben dürfen.) 
Die ${ }_{n} 38 / 42 \mathrm{er}$ " haben seit der Mitte bzw. seit Ende der 60er Jahre durch ihr strebsam-klagloses Funktionieren den Kultur- und Strukturwandel der alten Bundesrepublik kreativ mitgetragen, beeinflußt und nachhaltig stabil bewältigt und auf allen nur denkbaren Gebieten unter instabilen oder destabilisierten Verhältnissen für stabile Lebens- und Arbeitsumstände gesorgt. Sie taten dies, wie Blücher, Pfeil und Friedrichs richtig feststellten, mit einer gewissen Gelassenheit, die auf Erfolgserfahrung und -zuversicht beruhte, und mit jener Mischung von Kritikbereitschaft und Selbstsicherheit, die daraus resultierte, vor ${ }_{n} 68^{\prime \prime}$ geformt worden zu sein und nach " $68^{*}$ hinzugelernt und partielle Revisionen ihrer seitherigen Einstellungen und Verhaltensweisen vollzogen zu haben ${ }^{102}$. Das hat ihnen zu ihren Lebzeiten durch Adaptivität und Kreativität, gepaart mit Solidität, Flexibilität und Verläßlichkeit, zu Erfolg, Geltung und Ansehen verholfen, nicht nur ihnen selber, sondern auch den Institutionen, in denen sie gestaltend wirkten - bier mit einem eigenen "vitalen Akzent", auch ohne Gruppen- oder "Generationen"-Etikettierungen, nach denen sie übrigens auch selten gefragt wurden. Nach ihnen kamen "die 68er", und darauf folgte die "neue Unübersichtlichkeit" der Individualisierung (Habermas, Beck).

Neuerdings schlägt das Pendel wieder in die andere Richtung aus: die 14. ShellJugendstudie (2002) hat die erfolgsorientierten jungen Leute ${ }_{n} \mathrm{zwischen}$ pragmatischem Idealismus und robustem Materialismus" ausfindig gemacht ${ }^{103}$. So hätte man damals auch ganz treffend den Habitus der "38/42er " formulieren können, denn genau so brauchte man sie in den Strukturreformen seit der Mitte der 60er Jahre, und diese Einstellung scheint die beste Voraussetzung für die Gestaltung und Bewältigung der absehbaren Krisenszenarien zu sein.

"Generationalität" ist ein aufschlußreiches historiographisches Instrument, vor allem zur Markierung von Generationszusammenhängen und von differenten Generationseinheiten in ihnen. Neben den aktiven Generationseinheiten macht es vor allem die lebensgeschichtlich prägende Kraft von Milieus deutlich, in denen "Gesellschaft" sich über sich selbst verständigt. Und wenn im "Milieu" die „zündende Idee" wirksam wird, der "vitale Akzent" (Plessner), dann formiert sich eine "Generation". "Generationen" gestalten Geschichte, und der Gang der Geschichte gestaltet "Generationen“.

102 Lutz Niethämmer machte darauf aufmerksam, daß es nach „68“ Verzweigungen gab bezüglich der späteren sozial-kulturellen und politischen Einstellungen und Optionen der 42er“. In den akademischen Milieus bewegte sich die Mehrheit in Richtung Enthierarchisierung und "multikulturelle" Wissenschafts- und Umgangsformen, ohne jedoch die provokanten Regelverletzungen, Einschüchterungen und alle Aktionen, dem Ansehen der Universitäten zu schaden, gutzuheißen, geschweige denn sich an ihnen zu beteiligen. Als Willy Brandt dann "mehr Demokratie wagen" zur Regierungsmaxime erhob, wären viele vorher eben doch gern aktiv „dabeigewesen“, und so kam es zu den massenhaften nachträglichen Selbstzuschreibungen $\mathrm{zu} .68^{\circ}$ in den Jahren $1970 \mathrm{ff}$.

${ }^{103}$ Deutsche Shell (Hrsg.), Jugend 2002. Zwischen pragmatischem Idealismus und robustem Materialismus (Frankfurt a.M. 2002). 